\title{
METODOLOGIA PARA EL MODELAJE DE DATOS GEOGRAFICOS Y TOMA DE DECISIONES (MODAGE): UNA APLICACION A ESTUDIOS VULCANOLOGICOS
}

\author{
Luis Gmo Obando A. \\ Escuela Centroamericana de Geología, Laboratorio de Geomática, Apdo. 35-260, \\ Universidad de Costa Rica.
}

(Recibido 21/3/1997; Aceptado 20/10/1997)

\begin{abstract}
This paper proposes a methodology for Geographic Data Modeling and Decisions Making (MODAGE), with an application oriented to volcanological studies and decision making; due to the existence of data of international concern for the volcanological studies and to the importance of natural disasters provocated by the volcanic activity.

This methodology conduces the user through three logic levels until the previous implantation stage of its project, planned for a Geographic Information System (GIS). The levels are built from maps, photogeological maps, aerial photographs, satellite images, and other entries, which can be models of spatial representation of the reality, done by the matter expert. Consequently, as a starting point, the methodology requires such model.

Level 0, is the basic level (first step), of representation. Where the designer will show just the present graphic elements and their centroids, the centers of the graphics objects. Level 1, the external influences of other systems will be shown, this means, its environments. The systems that interact with the project to model will be represented. Level 2, the characteristics of the objects are defined and their spatial relations with other objects, its importance with respect to the hazards, crisis on natural disasters. At least, the detailed description of each modeled object is proposed by mean of a data dictionary. With the level 2. the system documentation is finished, and the project is ready to be implanted using a GIS. MODAGE is a methodology that has been designed with two purposes. First, the general character that documents the GIS database, describing the graphics objects and their attributes, and second, its helps to the poor structured decision making process during the occurrence of natural disaster or crisis, specialty those of volcanological type. MODAGE, is a new flexible tool, which leave open proposals for future research in this field.
\end{abstract}

RESUMEN: El presente trabajo propone una Metodología para el Modelaje de Datos Geográficos y Toma de Decisiones (MODAGE), con una aplicación orientada a estudios vulcanológicos y toma de decisiones; ello debido a la existencia de datos de interés mundial para los estudios vulcanológicos y a la importancia de la prevención de los desastres naturales provocados por la actividad volcánica.

Esta metodología conduce al usuario por 3 niveles lógicos hasta el paso previo a la implantación de su proyecto, planeado para un Sistema de Información Geográfico (SIG). Los niveles se construyen a partir de mapas topográficos, mapas geológicos, mapas fotogeológicos, fotos aéreas, de satélite, y otras entradas, los cuales pueden ser modelos de representación espacial de la realidad, realizado por el experto del ramo. Por lo tanto, como punto de partida, la metodología requiere de tal modelo.

Nivel 0, es el nivel básico de representación, en donde el diseñador solo mostrará los elementos gráficos presentes y sus centroides, los centros del objeto gráfico. Nivel 1, se mostrarán las influencias externas de otros sistemas, es decir, su entorno. Se representarán los sistemas que interactúan con el proyecto a modelar. Nivel 2, se definen las características de los objetos y sus relaciones espaciales con otros objetos, la importancia que estos tienen respecto a las amenazas, crisis o desastres naturales. Por último, se plantea la descripción detallada de cada objeto modelado, por medio de un diccionario de datos. Con el Nivel 2, se concluye la documentación del sistema, listo para ser implantado utilizando un SIG.

MODAGE, es una metodología que fue diseñada con dos propósitos. En primer lugar, de carácter general que documenta la base de datos SIG, describiendo los objetos gráficos y sus atributos, y en segundo lugar, asiste a la toma de decisiones poco estructuradas durante la ocurrencia de los desastres naturales o crisis, especialmente de tipo vulcanológico. MODAGE, es una herramienta nueva que deja abiertas propuestas a futuras investigaciones en este campo. 


\section{INTRODUCCIÓN}

Hasta el presente, dado que el interés de algunas compañías e investigadores de software, es el desarrollo y comercialización de sus productos relacionados con los Sistemas de Información Geográficos (SIG), muchos de éstos utilizan, para manipular datos, las bases de datos relacionales comerciales. Todos o casi todos los manuales de SIG describen al usuario cómo deben manipular su hardware, su software y cómo introducir sus datos, pero pocos manuales o publicaciones técnicas, indican cómo documentar su información, cómo el usuario diseñará su base de datos geográfica, y cuál será la relación de ésta con las tablas que almacenan datos no geográficos; esto es, datos alfanuméricos. El usuario no documenta cual fue el origen de los mapas, tablas, notas, datos de campo y sus correspondientes atributos; el usuario tiende a no registrar su planificación en cuanto a los niveles, colores, simbologías, grosores de líneas recomendables, análisis de los datos, etc.

El diseño de bases de datos alfanuméricos, está ampliamente desarrollado, documentado y en constante desarrollo (Modelo E-R -Entidad-Relación-, Modelo Semántico, Modelo Relacional de Codd, etc.), mientras que el modelaje de bases de datos geográficas parece ser un tema ignorado.

La metodología llamada Modelaje de Datos Geográficos (MODAGE), pretende esquematizar y describir las relaciones espaciales entre los objetos mostrados en un mapa, con el fin de que el diseñador pueda documentar e implantar su base de datos espacial. Con ello, se puede lograr una estandarización para futuros proyectos de SIG.

Es necesario aclarar que MODAGE es una propuesta nueva, descrita por primera vez en este trabajo y que como propuesta inicial y original, no está exenta de errores y omisiones. Por tanto, probablemente sea necesario realizar una labor posterior de pulimento para eliminar posibles fallas e inconsistencias. Por último, MODAGE, en este artículo, se orienta al dominio de la Vulcanología (una rama de la geología), sin embargo, la metodología puede ser utilizada en otras aplicaciones de índole general y tiene una especial aplicación a objetos puntuales.

Debido a que la cartografía, es una actividad tan corriente y difundida por todo el mundo, este modelo se basa en la representación de la realidad; esto es, un mapa, dado que los mapas tratan de representar la realidad en dos dimensiones, impresas ya sea en papel (nivel físico) o en un monitor.

La propuesta, está orientada a usuarios que se inician en el área de los Sistemas de Información Geográficos, es decir, usuarios finales, dado que los usuarios con experiencia en SIG (usuarios expertos) ya poseen y dominan una determinada metodología de trabajo. Sin embargo, esto no excluye en absoluto que el usuario experto, no esté exento de documentar su trabajo. Por tanto, esta propuesta también permite a dicho usuario la documentación de su SIG.

Dado que un mismo usuario podrá crear una gran gama de mapas y que cada uno dependerá de la disciplina científica, MODAGE pretende ser lo más flexible posible sin perder rigurosidad. Pretende elevar la representación física a un nivel lógico, más simplificado y fácil de usar, permitiendo al usuario visualizar la globalidad de los datos gráficos, sin perderse en los detalles propios de los mapas actuales.

Por último, se ha de señalar que MODAGE tiene una doble función:

a) ayuda a visualizar la globalidad de los datos gráficos y trascendiendo al usuario del nivel físico. Por otra parte, permite al usuario familiarizarse, dominar y madurar un proyecto SIG, en lo que respecta a los rasgos gráficos y los atributos relacionados con las entradas de datos, y

b) ayuda a la toma de decisiones en relación a las amenazas, crisis y desastres naturales.

\section{NIVELES DE REPRESENTACIÓN}

Las entradas pertenecientes a un determinado SIG están conformadas por tres grandes grupos de datos:

a) Los datos gráficos (geográficos, espacialmente ubicados en un sistema de coordenadas). Por ejemplo: mapas, puntos de muestreo, columnas estratigráficas, etc., que pueden ser tanto versiones finales (mapas impresos o editados previamente) o bien borradores procedentes de los datos de campo o sea mapas levantados en el 
campo, mapas fotogeológicos y geomorfológicos.

b) Tablas alfanuméricas, que representan datos de espesores, descripción de unidades geológicas, coordenadas de puntos de muestreo, etc., las cuales pueden tener la misma procedencia que los datos del punto anterior.

c) Sitios susceptibles a las crisis o amenazas naturales, tales como infraestructura civil, cultivos, poblados y ciudades, etc.

Los niveles de representación se definieron una vez realizado el estudio y análisis de las entradas, salidas, así como en la notoria necesidad de definir estándares y documentar el sistema de información. Todo sistema de información debe ser documentado, con el fin de registrar el origen de los datos y como material de consulta. De ese modo, cualquier cambio, será hecho lo más rápidamente posible y los analistas podrán localizar dichos cambios fácilmente. Los niveles de representación están diseñados para pasar de lo general a lo particular.

Dada la novedad de los SIGs, existe una fuerte tendencia de parte de los usuarios a trabajar sin documentar las entradas y salidas de la base de datos gráfica. Por lo tanto, al ocurrir un cambio de personal, estos no sabrán de donde se tomaron los datos, los mapas, los gráficos, y no podrá evaluar su confiabilidad. Este comportamiento, es muy similar al observado con cualquier sistema de información tradicional.

MODAGE propone 3 niveles según se aprecia en la figura 1 . Estos niveles van de lo general a lo específico y deben seguir el orden indicado en la figura 1. Los niveles se construyen a partir de los mapas fotogeológicos, geológicos, fotos aéreas, de satélite y otras entradas. Por lo tanto, como punto de partida, la metodología requiere del modelo espacial de datos.

Así, considérese los mapas basados en percepción remota (fotos aéreas, por satélite, radar, etc.), los cuales son relevantes para la toma de decisiones rápidas y además, considérese los mapas previamente confeccionados (mapas topográficos, mapas geológicos, etc.), como el modelo espacial de datos que deberá ser construido o esquematizado por el experto del ramo.

Es necesario aclarar que la metodología se ha diseñado pensando en todo momento en el concepto SIG, pero el software no hace su aparición sino hasta el final de la metodología (véase la figura 1 y 2) y es en ese momento cuando la metodología a cumplido su objetivo y llega a su fin, o sea, justo cuando el software entra en acción. Por otra parte, algunos términos usados en este trabajo son similares a los usados por algún SIG específico, pero estos términos pueden no coincidir con el concepto o significado que determinado SIG tiene para ese término.

\section{Nivel 0, General}

Nivel de base, nivel general, el más cercano al mundo real, en donde se muestran de una manera esquemática todos los objetos gráficos presentes y sus centroides, estos últimos, son puntos de referencia que representan el centro espacial del objeto y tienen como fin primordial el de generalizar los objetos a una forma más simple. Si el mapa es complejo en cuanto al número de objetos o entidades presentes, el usuario podrá considerar necesario la definición de varios subniveles 0 , según se representa en la figura 2 .

Es el nivel básico de representación, en donde el diseñador tan solo mostrará los elementos gráficos presentes (objetos). En este nivel, se definen los centroides (punto central del objeto), los cuales servirán de guía para representar las relaciones de la infraestructura civil, poblados, etc, con las amenazas naturales. El concepto centroide se introduce aquí con el único fin de generalizar y simplificar la representación gráfica. En este nivel, no se entra en detalle. El diseñador puede definir varios subniveles 0 . Los objetos pueden ser áreas, líneas, polígonos, arcos, puntos. Se contesta a la pregunta ¿cuáles elementos se van a diseñar? La figura $4 \mathrm{a}$, muestra el Nivel 0 obtenido del mapa geológico representado en la figura 3.

\section{Reglas:}

1) Dado que los Sistemas de Información Geográficos tienen la habilidad de representar los objetos en capas, no solo para facilitar el análisis sino también para facilitar la edición y reimpresión de los mapas, el diseñador debe procurar separar cada objeto en un nivel o capa diferente. Asigne, a cada objeto o grupo de objetos, una capa en la cual será representado y manipulado vía software. El diseñador deberá decidir los 
Fotogeología, Mapas, Datos de Campo, Tablas...

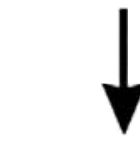

\section{Nivel 0}

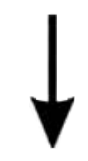

Nivel 1

Entorno

Diccionario de Datos
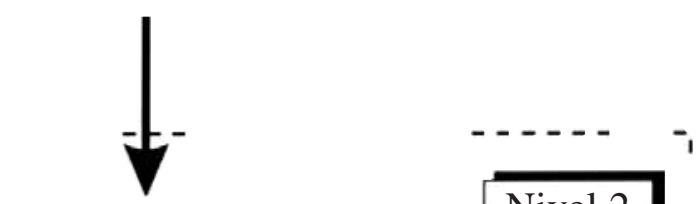

Nivel 2

\section{Relaciones}

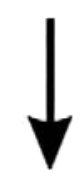

\section{Características} $\mathrm{y}$

Descripción

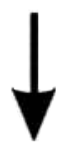

Implantación utilizando un SIG

Fig. 1. Metodología de Representación y Modelado de Datos Geográficos (MODAGE). 
Fotogeología, Mapas, Datos de Campo, Tablas...

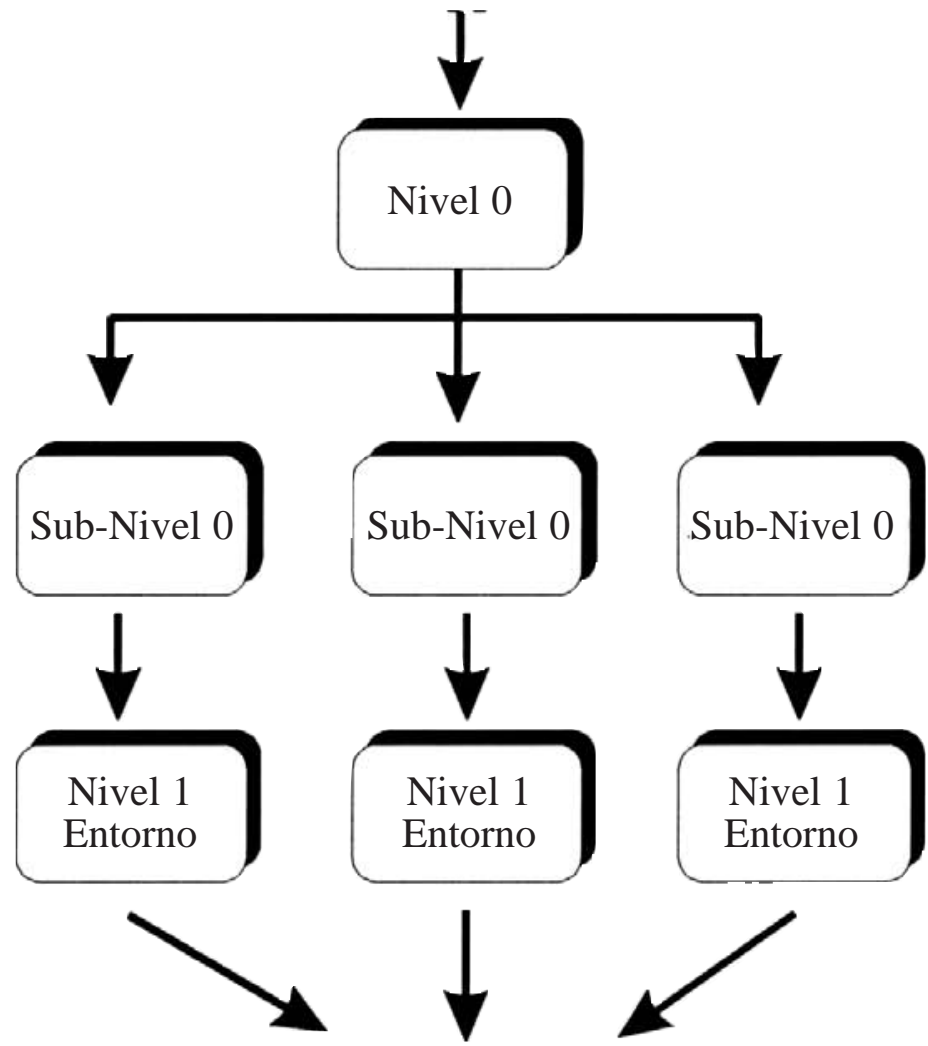

Diccionario de Datos

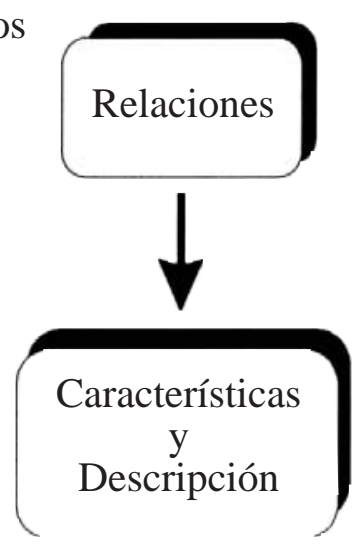

Nivel 2

'-

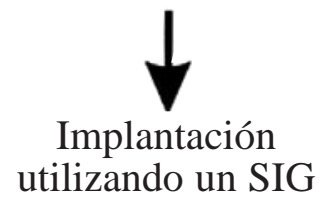

Fig. 2. Metodología de Representación de Datos Geográficos para Objetos Complejos. 
objetos que serán representados en determinada capa.

2) Es necesario que el diseñador determine los grados de pertenencia, para así poder definir la "capa base", esto es, el objeto que contiene a todos los demás. Por ejemplo, en un mapa que muestre una ciudad (cuadras o parcelas), señales de tránsito así como tomas de agua. La "capa base" será la ciudad, dado que en esta representación, la ciudad contiene a las señales de tránsito y contiene a las tomas de agua.

3) Dada la gran variedad de objetos que se pueden representar en un mapa, en todo momento, el usuario deberá especificar la simbología correspondiente utilizada para representar los objetos gráficamente. Para ello se puede utilizar los iconos estándar o simbologías más usados en geología.

4) $\mathrm{Si}$ un mapa posee un elevado grado de complejidad, el diseñador podrá considerar la posibilidad de definir uno o varios subniveles 0 (véase figura 2), con el fin de definir mejor la representación de las relaciones y su descripción detallada. La valoración de la complejidad de un mapa queda a juicio del experto o diseñador.

Por ejemplo, un nivel complejo puede ocurrir en un mapa cuando éste, presenta varios tipos de líneas (punteadas, dentadas, discontinuas, continuas) y varios tipos de grosores, o bien, diferentes tipos de arcos (dentados, lisos, etc).

\section{Del Centroide:}

Algunos SIGs, utilizan el centroide como punto de unión entre un objeto y sus atributos no gráficos, por ejemplo, fechas, nombres, texto, etc, unidos a polígonos o líneas. Para MODAGE, los centroides al igual que sus relaciones (véase más adelante "Definición de Relaciones") tienen como fin primordial el de generalizar los objetos a una forma más simple, y permitir gráficamente (como esquema), establecer las relaciones espaciales entre los objetos que se encuentran bajo alguna amenaza, por ejemplo: poblados, ciudades, infraestructura civil, cultivos, etc. y el objeto que causa la amenaza. Para nuestro caso de aplicación, los objetos causantes de amenazas naturales, serán los volcanes, sus cráteres activos u algún otro fenómeno volcánico relacionado, y los objetos bajo amenaza, serán todos aquellos objetos susceptibles de sufrir daños durante las manifestaciones volcánicas.

La finalidad del centroide no es más que un recurso que ayuda al diseñador en la generalización de los objetos, con el fin de bajar la complejidad y lograr la abstracción del nivel físico, dado que con el punto (centroide), la representación reduce su dificultad y adquiere claridad (compárese la figura 3 con la figura 4a). Por último, es necesario aclarar que el diseñador puede utilizar la definición de centroides, para aplicaciones que no necesariamente tengan que ver con las amenazas naturales, siempre y cuando no causen confusión.

\section{Reglas:}

1) Para cada objeto, sin importar su forma, defina, tan solo por inspección visual, un centroide, esto es, un punto central, a partir del cual se establecerán las relaciones. Represente el centroide con un círculo pequeño relleno en color negro.

2) Identifique (rotule) el centroide asignándole un número o letra, se sugiere que la identificación sea acompañada de una letra y un número que represente el objeto y el número de capa en la cual será mapeado. Por ejemplo se tienen:

$$
\begin{aligned}
& \text { A1 = OBJETO A, CAPA } 1 . \\
& \text { B16= OBJETO B, CAPA } 16 . \\
& \text { C } 9=\text { OBJETO C, CAPA } 9 .
\end{aligned}
$$

3) Basta con determinar un centroide para los objetos de la misma clase o del mismo tipo. Para ello, el diseñador escogerá el objeto más representativo o el más relevante para su estudio. Por ejemplo, si en un complejo volcánico existen 5 cráteres, uno de los cuales está activo actualmente, el diseñador podrá escoger el cráter activo para establecer sus relaciones, dado que éste puede ser el más importante. Sin embargo, si es necesario descomponer el nivel 0 en varios subniveles 0 , se hace necesario asignar un centroide para cada componente del subnivel. Por ejemplo, el conjunto "cráteres" puede ser formado por los siguientes miembros: cráter activo, cráter adventicio, cráter inactivo, etc. Si el diseñador desea indentificar cada tipo de cráter, deberá asignar un centroide a cada miembro en algún subnivel. Un segundo ejemplo, puede ser el conjunto de Fallas Geológicas, las 
cuales se pueden dividir en miembros llamados, fallas inversas, fallas normales y fallas transcurrentes, si todas están presentes en un mapa, entonces se deberá asignar un centroide a cada una en un determinado subnivel.

Si el objeto en cuestión está muy disperso en el mapa, el centroide será definido para el objeto de la misma clase (o del mismo tipo) con mayor área, siguiendo los criterios anteriores. Si del todo no es posible obtener un centroide, por ejemplo, una red de carreteras o una red de drenaje (ríos) muy compleja, el diseñador podrá optar por una representación arbitraria o del todo no representarla, siempre y cuando se indique la dificultad.

5) Construya un mapa esquemático de centroides. Si desea, puede representar la forma del objeto esquemáticamente. La figura 4a, muestra el Nivel 0, con todos los objetos encontrados en el mapa de la figura 3. Obsérvese la documentación de los objetos por medio de la simbología especificando el icono, la capa y la descripción.

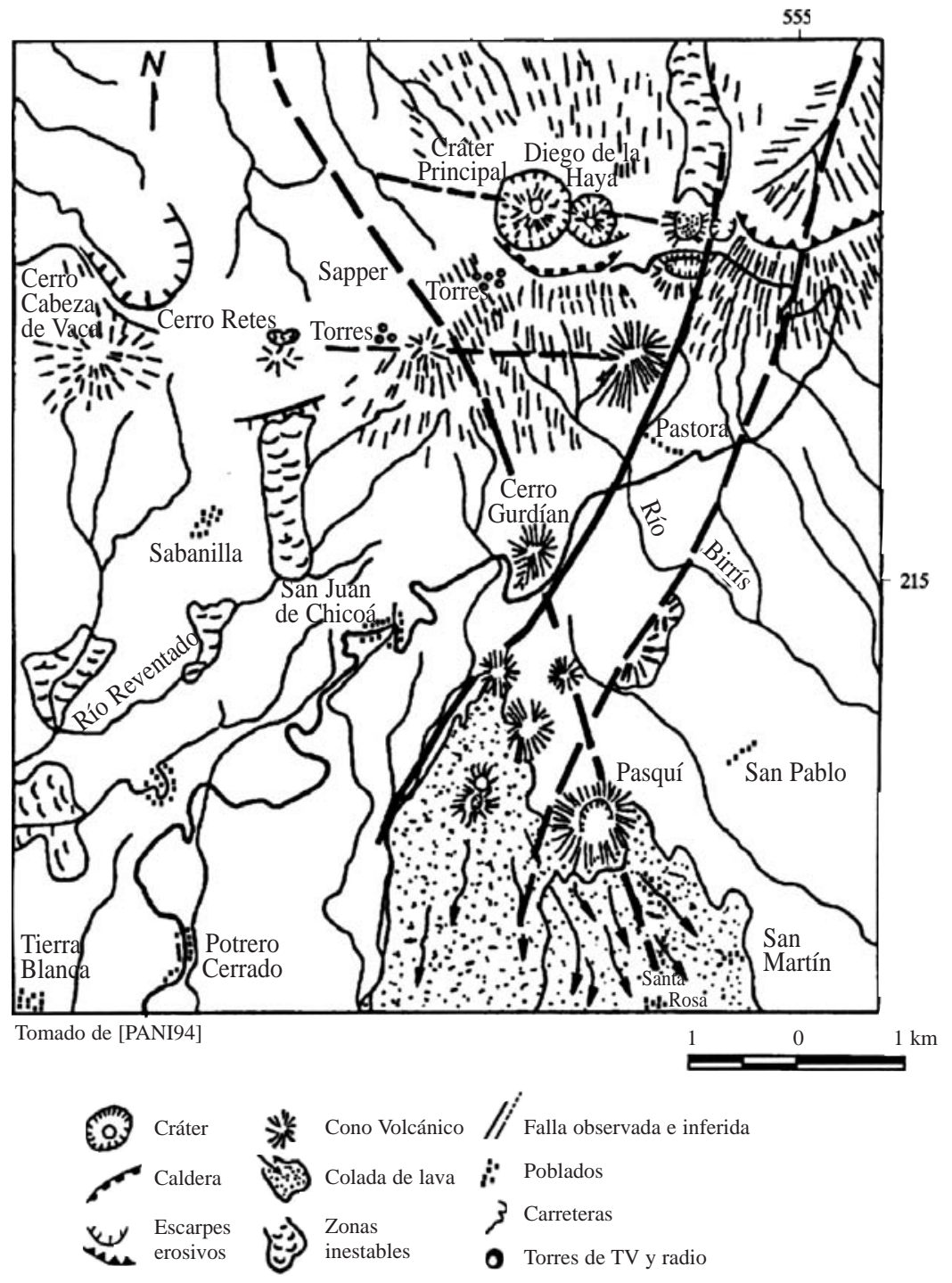

Fig. 3. Mapa geomorfológico del Volcán Irazú. 


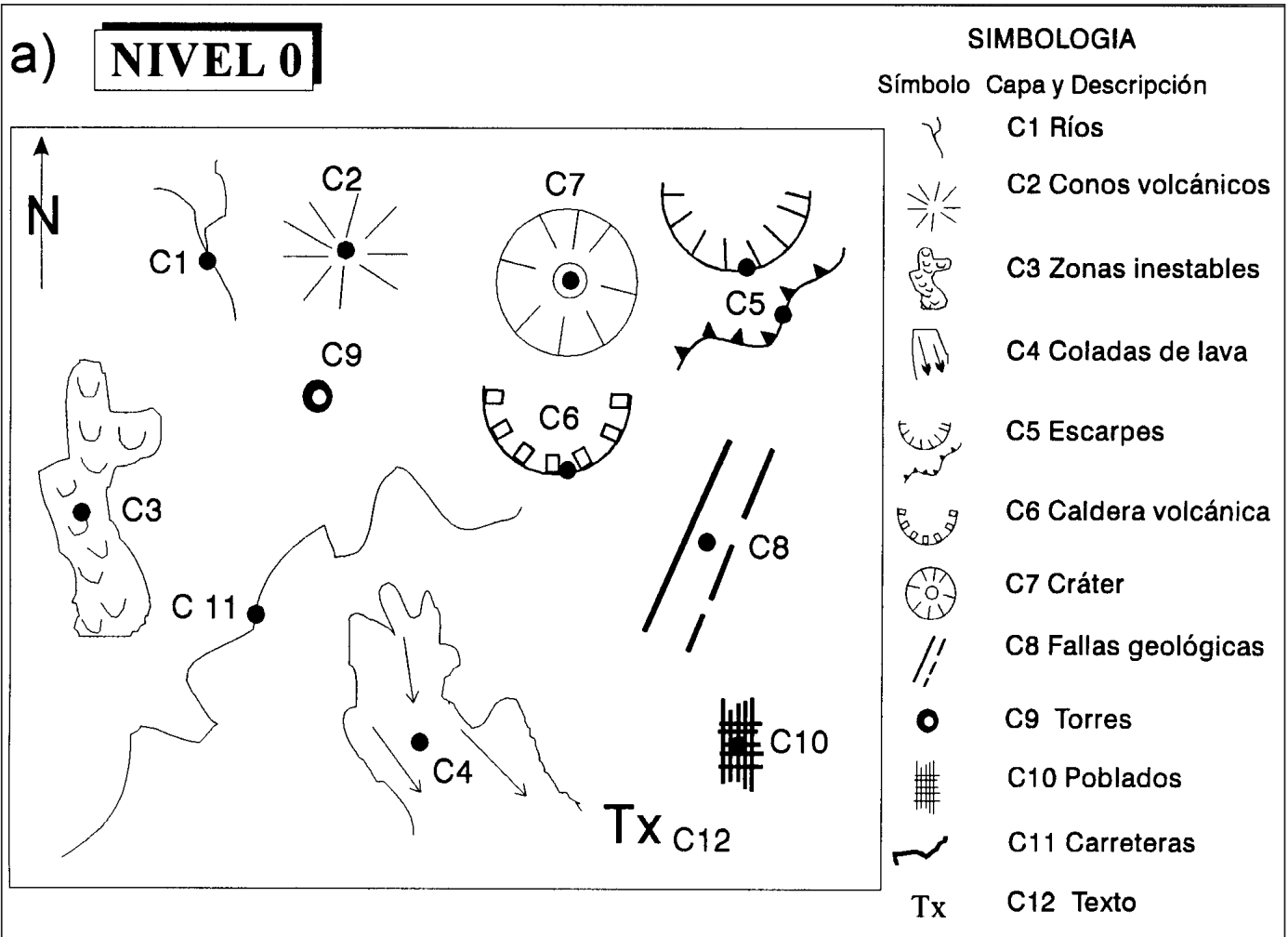

b) NIVEL 1

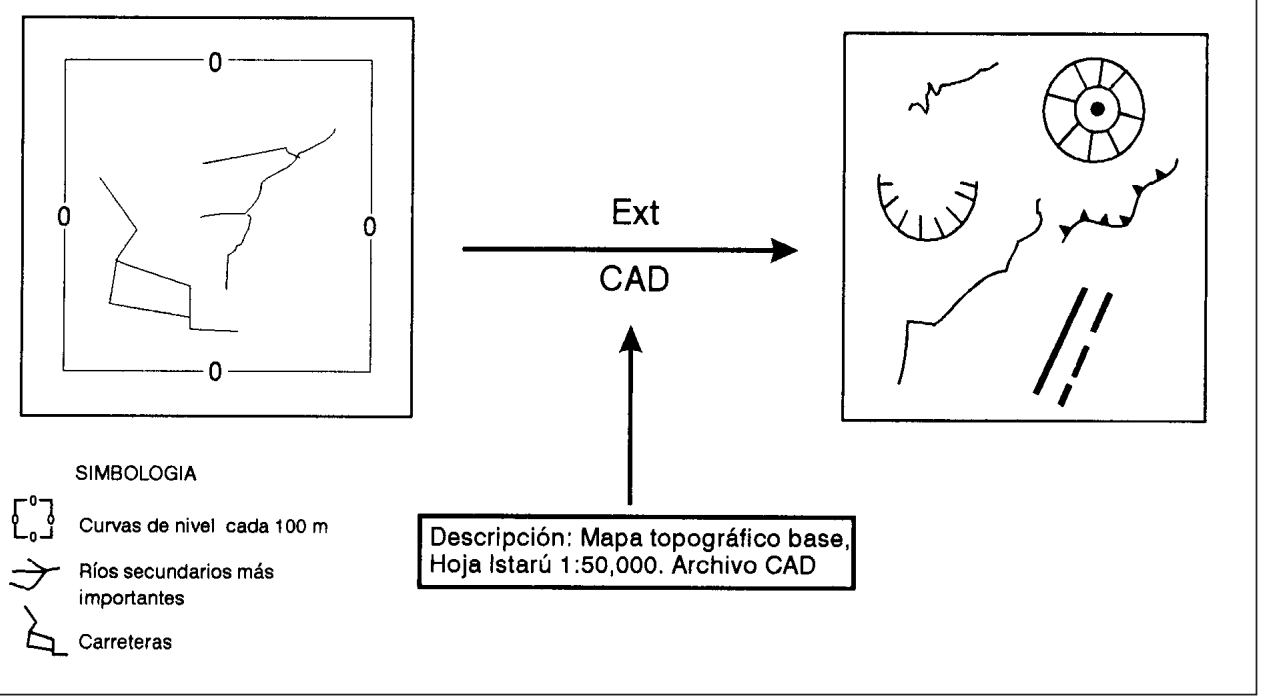

Fig. 4. Nivel 0 (general) y Nivel 1. a) Nivel 0 a partir de la figura No. 3, note que en este nivel solo se muestran los elementos presentes y los cenroides. b) Nivel 1, interacción de los Sistemas Externos. En este caso un CAD. 


\section{Nivel 1, Entorno}

En este nivel, se muestran las influencias externas de otros sistemas, es decir, su entorno. Así, se representarán los sistemas que interactúan con la base de datos gráfica. Por ejemplo, se representará la toma de datos alfanuméricos o gráficos a partir de un CAD, un Sistema Administrador de Bases de Datos (SABD) u otro SIG y qué tipo de datos se tomarán de esos archivos, por ejemplo, topografía, datos puntuales, ríos, carreteras, etc. (Fig. 1, para subniveles véase la figura 2).

En este nivel se muestra el entorno (contexto), las influencias externas de otros sistemas, por ejemplo, SABD, mapas topográficos, sensores remotos, importación de datos de otro SIG, etc. Se contesta a la pregunta ¿qué sistemas interactúan o pueden interactuar con el actual? Con este nivel, se documenta la procedencia de los datos externos al sistema diseñado. La filosofía de este nivel es pensar en los objetos que hacen falta para completar o complementar el Nivel 0.

\section{Reglas:}

1) Dibújese esquemáticamente todos los sistemas del entorno que influencian al Nivel 0 . Por ejemplo, curvas de nivel, sistemas de drenaje, topografía en general, tablas, etc. Indicar la simbología respectiva.

2) Dibújese una flecha dirigida hacia lo que será el Nivel 0. En la parte superior rotularla con la abreviatura "EXT" de externo, en la parte inferior el sistema o sistemas de donde proceden los datos (CAD, SIG, etc, figura $4 b)$.

\section{Nivel 2, Definición y Características}

En este nivel, se definen las características de los objetos, sus relaciones con otros objetos, la importancia que estos tienen respecto a las crisis, amenazas y desastres naturales; por último, se plantea la descripción detallada de cada objeto modelado. Con el Nivel 2, se concluye la documentación del sistema. La descripción detallada, viene siendo el Diccionario de Datos del proyecto SIG, dado que en este último paso, se detallan las entidades involucradas en el sistema.
Seguidamente, se definirá cada nivel utilizando para ello un ejemplo real (Fig. 3). Este ejemplo es tomado de la cartografía del área del Volcán Irazú (PANIAGUA et al., 1993). Observando el mapa, el lector puede notar su complejidad y la variedad de rasgos representados en él. Por tanto, para darse a entender y no crear ambiguedad, el experto geólogo o vulcanólogo debe colocar la respectiva simbología.

Corresponde al último nivel de diseño, en donde se muestran sus relaciones y la descripción detallada para cada clase de objeto y sus variaciones. Este nivel viene siendo el Diccionario de Datos del proyecto SIG. Se contesta a las preguntas iidentificación de los objetos, cuántos hay, formas y símbolos de esos objetos, relaciones espaciales? (véase la figura 1 y 2).

El Nivel 2, se representa utilizando iconos y flechas de asociación, las cuales no son vectores matemáticos ni flujos de datos. Estas flechas tan solo señalan las relaciones y los valores que la describen. El Nivel 2 se compone de 2 pasos: definición de relaciones (o asociaciones) y caracterización y descripción detallada de cada objeto.

Paso 1: Definición de Relaciones o Asociaciones, aquí se usará el término relación como sinónimo de asociación o enlace. El paso 1 define las relaciones o enlaces entre cada objeto y su medio: Objeto relación objeto, Objeto relación otro objeto, Objeto relación medio. Se contesta a la pregunta ¿cuáles son las relaciones entre los objetos?

Las figuras $5 \mathrm{a}, 5 \mathrm{~b}, 5 \mathrm{c}$ y $5 \mathrm{~d}$, muestran ejemplos de asociaciones entre 2 objetos de la misma clase o bien objetos de diferente clase. Se asocian objetos simples y complejos.

Como se indicó anteriormente, el fin único de las relaciones y sus centroides es el de representar fácilmente las crisis o amenazas naturales que afectan o podrían afectar un determinado objeto, sea éste, infraestructura civil, asentamientos humanos, parques nacionales, etc; cualquier objeto que el usuario considere importante y suceptible de ser amenazado y ponga a juicio su existencia por algún fenómeno natural.

Esta representación, será especialmente conveniente al mostrar esquemáticamente las relaciones espaciales entre los objetos amenazados 
1) RELACION O ASOCIACION DE A SOBRE A: A R A

1a) Cercanía Variable: a partir del centroide " $A$ ", otro objeto " $A$ " se encuentra cercano por la derecha,a una distancia variable
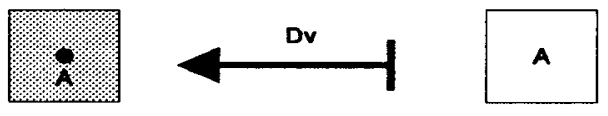

1b) Cercanía Absoluta: a partir del centroide " $A$ ", otro objeto " $A$ " se encuentra cercano, a 10 unidades por la derecha.
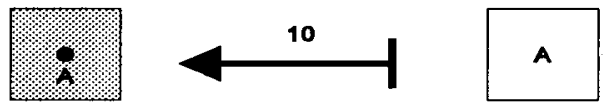

1c) Lejanía Variable: a partir del centroide "A", otro objeto "A" se encuentra lejano por la derecha, a una distancia variable.
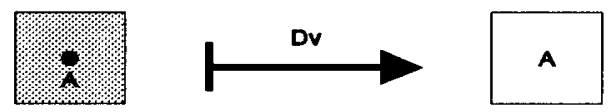

1d) Lejanía Absoluta: a partir del centroide " $A$ ", otro objeto "A" se encuentra lejano por la derecha, a una distancia de 10 unidades.
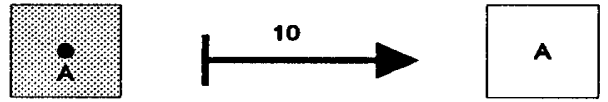

2) RELACION O ASOCIACION DE A SOBRE B: A R B

2a) Cercanía Variable: a partir del centroide "A", otro objeto "B" se encuentra cercano por la izquierda.
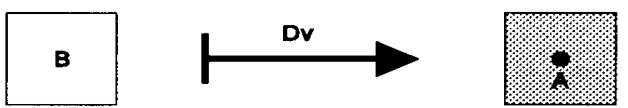

2b) Cercanía Absoluta: a partir del centroide " $A$ ", otro objeto " $B$ " se encuentra cercano, a 20 unidades por la izquierda.
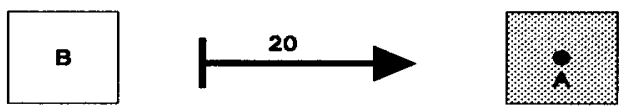

2c) Lejanía Variable: a partir del centroide "A", otro objeto "B" se encuentra lejano por la izquierda, a una distancia variable.
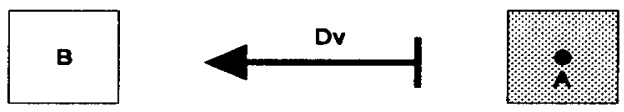

Fig. 5a: Ejemplos de relaciones o asociaciones. 
2d) Lejanía Absoluta: a partir del centroide "A", otro objeto "B" se encuentra lejano a 20 unidades por la izquierda,

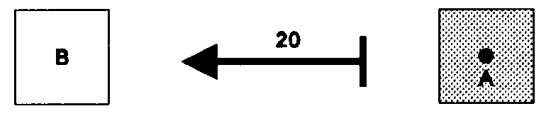

3) REPRESENTACION DE GRADOS

3a) Grados: a partir del objeto "A", existen dos objetos lejanos "B" y "C", ubicados a 50 grados y a 90 grados desde "A", respectivamente. El objeto "B", se encuentra a 10 unidades y el objeto "C" a una distancia variable respecto a "A".

En este caso, el objeto " $B$ " se encuentra al N 50 E respecto a "A". El objeto "C" se encuentra totalmente al este (E) respecto a " $A$ ".

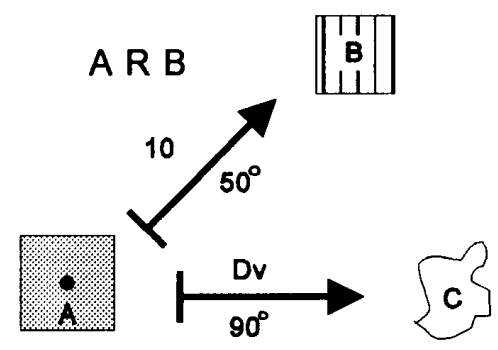

A R C

\section{4) TIPO DE OBJETO RELACIONADO}

4a) Objeto compacto: Todos los objetos anteriores corresponden a objetos compactos

4b) Objeto Atomizado o Esparcido: A partir del objeto compacto "A" (centroide), existen tres objetos "B", 2 de ellos son compactos y el tercero está atomizado en un área que forma un arco de 30 grados respecto a "A".La asociación denota una relación de lejanía y una distancia variable.

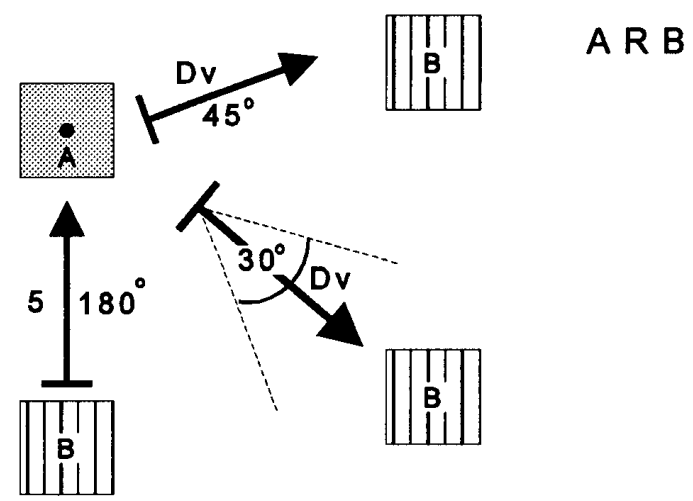

Fig. 5b: Ejemplos de relaciones (continuación). 


\section{TIPO DE VECINDAD}

Descripción:

Símbolo:

Distancia Variable:

Dv

Distancia Absoluta:

(basada en la escala del mapa y

$10,200,1,9$

especificada por el usuario)

Cercanía Variable:

Cercanía Absoluta:

(basada en la escala del mapa)

Lejanía Variable:

Lejanía Absoluta:

(el valor está basado en la escala del mapa y la distancia que separa el objeto del centroide)

Cercanía Inmediata
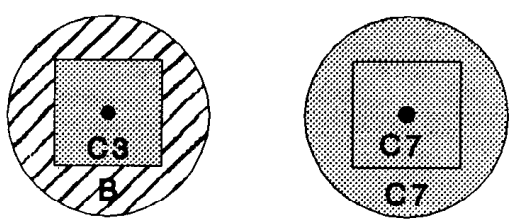

Objeto Reflexivo

\section{REPRESENTACION DE GRADOS}

(N)orte

$0,360^{\circ}$

Orientación:

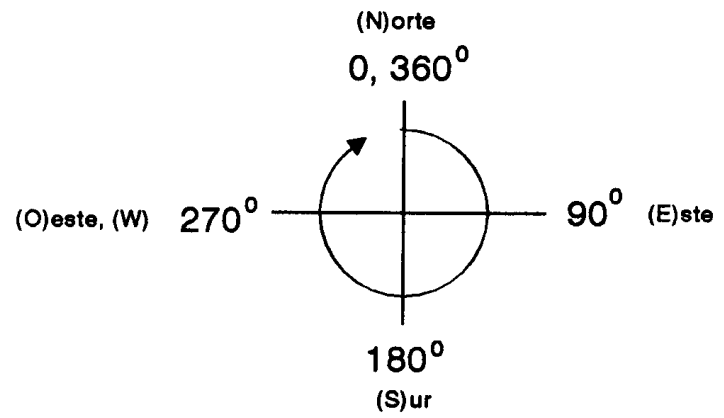

Grados Absolutos:

Grados Variables (gv):

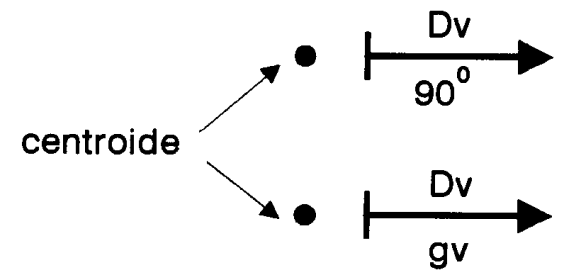




\section{TIPO DE OBJETO RELACIONADO}

Objeto Compacto:

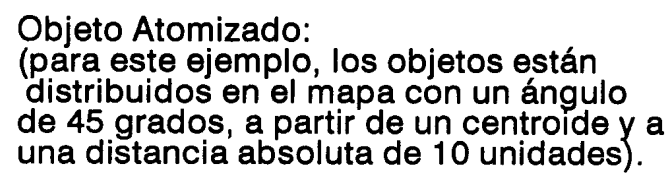

Las unidades serán especificadas por el diseñador.
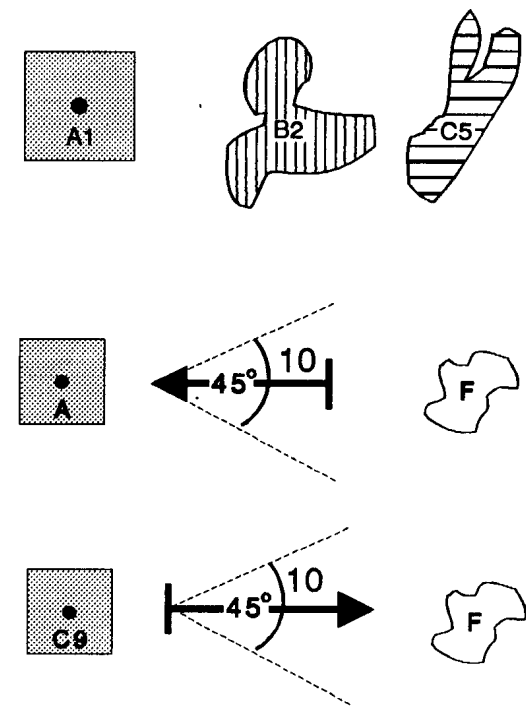

Fig. 5d: Símbolos de vecindad (continuación).

en casos de emergencia. Será el experto del ramo quién decidirá qué objeto está o estará mayormente amenazado. Creemos que esta representación es útil para:

1) La toma de decisiones rápidas, durante las emergencias (al fase del "Durante") o bien durante las fases del "Antes" (prevención y mitigación). El diseñador podrá dibujar estas relaciones para ser mostradas a todas aquellas personas que tienen poder de decisión, sin necesidad de esperar a que la versión final o inicial del mapa de campo sea confeccionado.

2) Para aquellos casos en donde el recurso computacional, así como el software, sea escaso o bien el equipo de cómputo ha sufrido algún daño durante la emergencia, por ejemplo, terremoto.

3) Documentar y crear un estándar, como ya se anotó anteriormente, de la Base de Datos Geográfica.

Para la aplicación que nos ocupa, y para objetos puntuales importantes, como por ejemplo: ciudades, poblados, hospitales, puentes, represas, tomas y tanques de agua potable, etc.; en cualquier momento, la representación podrá ser ubicada espacialmente sin utilizar el mapa, tan solo, señalando las coordenadas del cráter del volcán (centroide), dado que todas las relaciones se construyen con referencia al cráter activo o el objeto generador del fenómeno volcánico. $\mathrm{O}$ incluso, con la actual tecnología para ubicación espacial por satélite (Sistema de Posicionamiento Global o GPS, del inglés Global Positioning System), se puede determinar las coordenadas espaciales de cualquier objeto sin utilizar un mapa topográfico. Siendo así, cualquier objeto puntual puede servir para la construcción de la relación.

En lo que se refiere a objetos no puntuales como áreas, carreteras, ríos, etc., lo anterior puede no aplicarse (en dependencia de su escala), dado que el centroide por sí mismo, es insuficiente para definir el objeto completamente, para ello, se requiere de un modelo a escala (mapa o esquema) o bien suministrar la coordenadas espaciales del objeto, con el fin de lograr definirlo vía software.

\section{Reglas:}

De las relaciones o asociaciones

1) Toda relación será definida de acuerdo a los centroides del Nivel 0 . 
2) Las relaciones serán definidas para cada objeto amenazado, representado en el Nivel 0 . El usuario decidirá, según el contexto, cual relación será equivalente. Por ejemplo, si se define la relación "c4" en "d7" (denotado c4 R d7), el usuario decidirá si es equivalente a "d7 R c4".

3) Al menos será representada una relación para cada objeto de la misma clase, el objeto más representativo, será relacionado siempre. Sin embargo, el diseñador podrá representar tantas relaciones como desee, empezando desde el objeto de la misma clase más importante, hasta el objeto de la misma clase menos importante.

4) El mapeo de las relaciones se hará de la siguiente manera (véase la figura $5 \mathrm{c}$ ):

a) El norte geográfico será ubicado en la parte superior del mapa o esquema (Nivel 0), esto para definir la orientación en grados de las relaciones con los centroides. Los grados serán tomados siguiendo las manecillas del reloj, así el este geográfico (E) estará a $90^{\circ}$ respecto al norte geográfico, el sur geográfico (S) a $180^{\circ}$ respecto al norte, el oeste $(\mathrm{O}$ o W) estará a $270^{\circ}$ respecto al norte. $0^{\circ}$ o $360^{\circ}$ representan el norte $(\mathrm{N})$.

b) La orientación de un objeto respecto a otro, será dada por una flecha entrante o saliente al objeto en cuestión, que contendrá: los grados y su distancia, la cual puede ser a escala. Tanto los grados como las distancias pueden ser absolutas o variables (Fig. 5c, 5d). La medida de cercanía o lejanía de un centroide respecto a otro, será dada por la escala del mapa u otra escala que el usuario deseé definir, siempre y cuando se especifique claramente. Se sugiere que use la misma escala del mapa a modelar con el fin de ser congruente con el mismo. Se debe aclarar que las flechas no son vectores ni flujos de datos, son iconos utilizados para establecer la relación o asociación con los objetos, por tanto, no necesariamente deben ser hechos a escala.

La figura 6, muestra las relaciones (C7 R C10) del Volcán Irazú (C7), con respecto al sitio bajo amenaza (C10). El lector notará que los cuadrados rodeando los poblados representan el tipo de amenaza (máxima, alta, moderada) de acuerdo a los estudios geológicos (PANIAGUA, 1994). Si la amenaza es relativamente inexistente o baja, el cuadrado se omite.

Por otra parte, si el usuario lo desea, se podrá especificar con más detalle el tipo de amenaza sobre cada poblado, por ejemplo, caída de cenizas, bloque, lavas, etc.

Como ejemplo, la relación C9 (Torres) en C7 (Volcán Irazú), debe entenderse así: con respecto a C7, las torres de televisión y radio están muy cercanas (flecha entrante a C7), es decir, a $0,85 \mathrm{~km}$ y a $215^{\circ}$ (al SW-W) respecto al cráter. Para este caso, la amenaza es máxima.

La relación $\mathrm{C} 10$ en $\mathrm{C} 7$, o sea, el poblado de Tierra Blanca (C10) con respecto al Volcán Irazú (C7), define una relación de lejanía (relativamente, flecha saliende de $\mathrm{C} 7$ ), el poblado se localiza a $8,8 \mathrm{~km}$ y al $211^{\circ}$ del cráter. De acuerdo a los mapas actuales de amenaza, este poblado no se encuentra amenazado, por ello no aparece un cuadrado. Por otra parte, las flechas de relación son salientes para acentuar la relación de lejanía.

En la figura 5c, se representan los símbolos de vecindad para señalar: distancia variable entre objetos, distancia absoluta entre objetos, grados variables entre objetos, grados absolutos entre objetos, cercanía absoluta entre objetos, cercanía variable entre objetos, lejanía absoluta entre objetos, lejanía variable entre objetos, objeto compacto u objeto esparcido (atomizado) .

Paso 2: Característica y Descripción detallada de cada objeto. Este paso será el previo a la implantación de cada objeto, vía software. Se hará una descripción detallada para cada clase de objetos. Esta fase es importante dado que al ser documentada la representación y el modelo, se confecciona una buena guía para crear la base de datos geográfica. Por tanto, con este último paso se confecciona el diccionario de datos del proyecto, al definir y detallar cada entidad.

\section{Reglas:}

1) Separe los objetos en grupos o meta-entidades, usualmente todos lo objetos caen dentro de alguna de estas categorias, por ejemplo: 


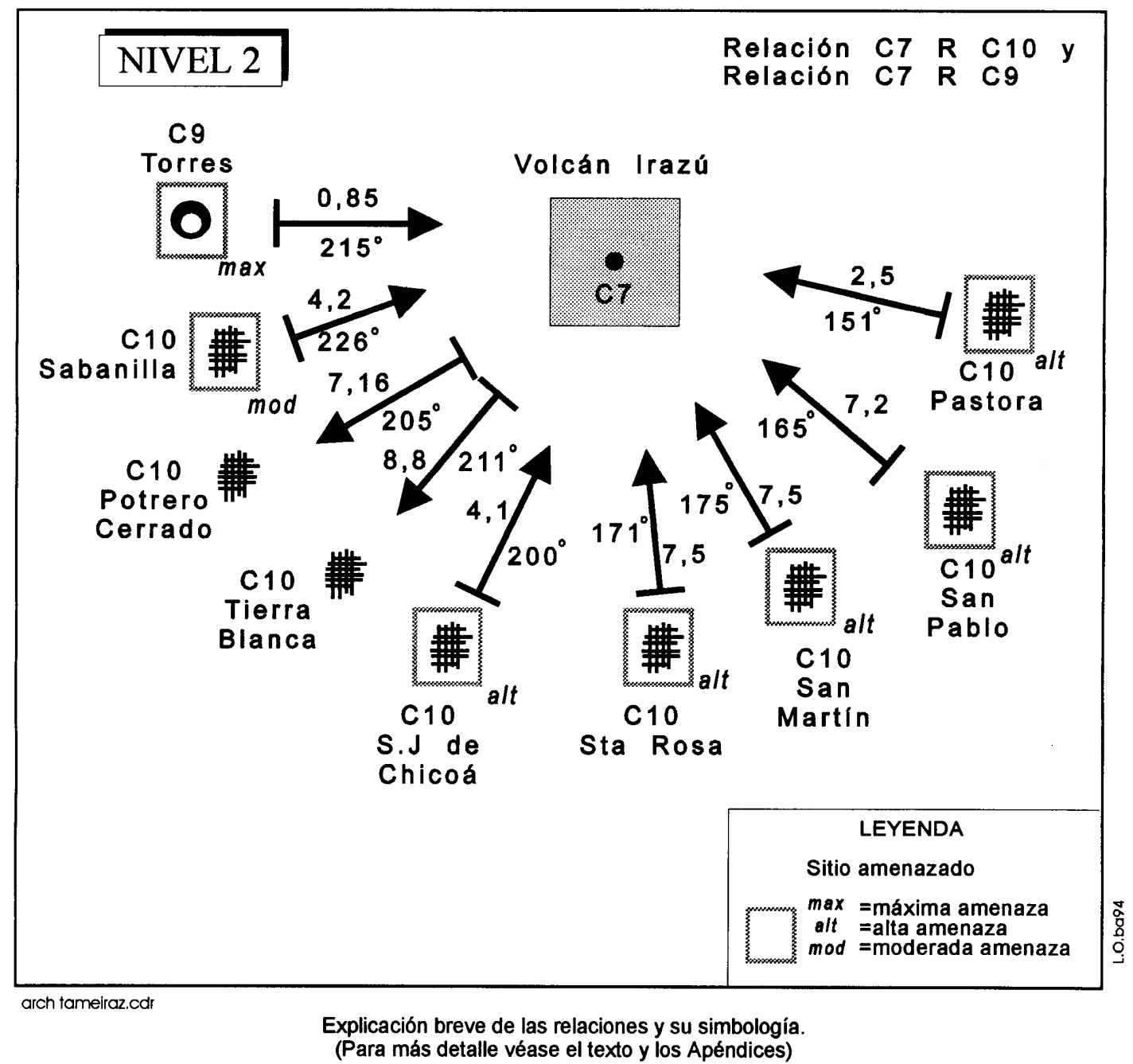

Relación de Cercanía:

Con respecto a $\mathrm{C} 7$, el objeto $\mathrm{C9}$ se encuentra bajo amenaza máxima. C9 se encuentra a 0,85 km de acuerdo a la escala del mapa y a 215 grados de azimut respecto a $\mathrm{C} 7$.

La flecha denota tan solo la relación de cercanía.

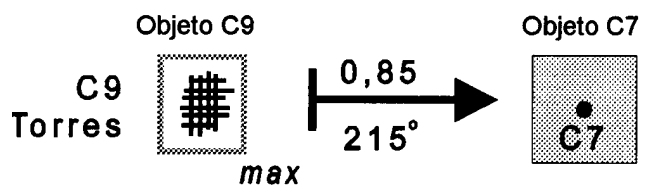

Relación de Lejanía:

Con respecto a $\mathrm{C} 7$, el objeto $\mathrm{C} 10$, está lejano sin amenaza. C10 se encuentra a 2,5 km de acuerdo a la escala del mapa y en este caso a 90 grados de azimut.

La flecha denota tan solo la relación de lejanía.

Objeto $\mathrm{C7}$

Objeto C10

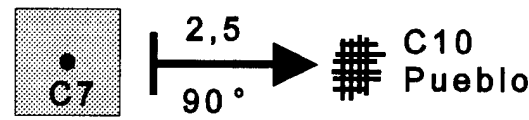


Meta-entidad: Líneas.

Meta-entidad: Areas.

Meta-entidad: Texto.

Meta-entidad: Puntos.

2) Cada meta-entidad debe contener la siguiente descripción:

\section{Meta-entidad Líneas:}

- Nombre de la META-ENTIDAD: Se especifica el nombre del la Meta-entidad, Líneas en este caso.

- Nombre de la ENTIDAD: Se especifica si es carretera, río, contorno o curva de nivel, etc., con su símbolo en el mapa.

- COORDENADAS X y $Y:$ : Indica las coordenadas para definir la línea, si éstas son manuales. Si son digitalizadas directamente, no es necesario. Sin embargo, para documentar su posición espacial deben indicarse.

- CANTIDAD: Indica el número total de estos objetos presentes en el mapa que representa ese objeto en una determinada capa.

- CAPA: Indica el número de capa en donde el objeto se representará $(2,4,5$, 89).

- COLOR: Indica un color de la paleta de colores.

- TIPO: Tipo de línea, continua, discontinua, punteada, etc.

- GROSOR: Indica los milímetros de grueso de la línea.

- SIMBOLO: Indica el símbolo usado para su representación.

- VALOR MINIMO Y MAXIMO: Si son líneas de isovalores se deben indicar los valores mínimo y máximo así como su incremento.

- PROCEDENCIA: Indica su origen, ya sea de otro sistema, del análisis de mapas o dato de campo.
- DESCRIPCION: Cualquier comentario que ayude a una definición más completa del objeto. Ejemplo: "Topografía base, 1:50.000, Hoja Arenal, solo representar las curvas de nivel cada $100 \mathrm{~m}$ y los ríos principales".

\section{Meta-entidad Areas:}

- Nombre de la META-ENTIDAD: Se especifica el nombre de la Meta-entidad, Areas en este caso.

- Nombre de la ENTIDAD: Se especifica si es lago, ciudad, área de manglar, área de lavas o piroclástos, etc., con su símbolo en el mapa.

- COORDENADAS X y Y: Señale las coordenadas manualmente para definir el área. Si las áreas son complejas y se usa un digitalizador, no es necesario indicarlas. Sin embargo, para documentar su posición espacial deben indicarse.

- CANTIDAD: Indica el número total de estos objetos presentes en el mapa.

- CAPA: Indica el número de capa a representarse $(2,4,5)$.

- COLOR: Indica un color de la paleta de colores.

-FORMA: Indica si es ovalada, cuadrada, circular, lobulada, tipo amiba, abierta o cerrada, etc.

- RELLENO: Indica si es de algún color, hachurado y su inclinación, etc.

- TIPO DE BORDE: Tipo de línea, continua, discontinua, punteada, etc.

- GROSOR: Indica los milímetros de grueso de la línea de contorno del polígono.

- SIMBOLO: Indica el símbolo usado para su representación, tipo de hachurado.

- PROCEDENCIA: Indica su origen, ya sea de otro sistema, del análisis de mapas o dato de campo. 
- DESCRIPCION: Cualquier comentario que ayude a una definición más completa del objeto.

\section{Meta-entidad Texto:}

- Nombre de la META-ENTIDAD: Se especifica el nombre de la Meta-entidad, Texto en este caso.

- Nombre de la ENTIDAD: Se especifica si es nombre de lago, ciudad, área de manglar, área lavas, texto isovalor, etc.

- COORDENADAS X y $Y$ : Indique las coordenadas manualmente para definir la posición del texto para un punto central del texto. Si son digitalizadas directamente, no es necesario. Sin embargo, para documentar su posición espacial deben indicarse.

- CANTIDAD: Indica el número total de estos objetos presentes en el mapa.

- CAPA: Indica el número de capa a representarse.

- COLOR: Indica un color de la paleta de colores.

- TIPO: Tipo: roman, courier, helvética, etc.

- GROSOR: Indica los milímetros de grueso o bien el tamaño en puntos.

- SIMBOLO: Indica el símbolo usado para su representación, caso de ser poco común.

- PROCEDENCIA: Indica su origen, ya sea de otro sistema, del análisis de mapas o dato de campo.

- DESCRIPCION: Cualquier comentario que ayude a una definición más completa del objeto.

\section{Meta-entidad Puntos:}

- Nombre de la META-ENTIDAD: Se especifica el nombre del la Meta-entidad, Puntos en este caso.

- Nombre de la ENTIDAD: Se especifica si es punto de muestreo, cráter, pozo, fuente de agua, etc.
- COORDENADAS X y Y: Indica las coordenadas manualmente para definir el punto. Si son digitalizadas directamente, no es necesario. Sin embargo, para documentar su posición espacial deben indicarse.

- CANTIDAD: Indica el número total de estos objetos presentes en el mapa.

- CAPA: Indica el número de capa a representarse $(2,4,5)$.

- COLOR: Indica un color de la paleta de colores.

- SIMBOLO: Indica el símbolo usado para su representación.

- RELLENO: Indica si es rellenado en algún color, hachurado y su inclinación. etc.

- GROSOR: Indica los milímetros de grueso

- PROCEDENCIA: Indica su origen, ya sea de otro sistema, del análisis de mapas o dato de campo.

- DESCRIPCION: Cualquier comentario que ayude a una definición más completa del objeto.

Continuando con el desarrollo del ejemplo, el siguiente paso es mostrar los detalles de cada objeto, categorizado por su meta-entidad. La descripción detallada procede del Nivel 1 de la figura $4 \mathrm{~b}$ y de las relaciones mostradas en la figura 6 . En este ejemplo, se muestran solo algunos de estos objetos. (Tablas $\mathrm{N}^{\mathrm{o}} 1 \mathrm{a}$ )

Ejemplo: NIVEL 2, descripción detallada de cada objeto por meta-entidad

Aquí, se definen las respectivas meta-entidades con tan solo algunos objetos:

Meta-entidad:

AREAS $=\mathrm{C} 3$ y C4, LINEAS $=\mathrm{C} 8$ y C11,PUN TOS $=\mathrm{C} 7, \mathrm{TEXTO}=\mathrm{C} 12$. 


\begin{tabular}{|c|c|c|}
\hline 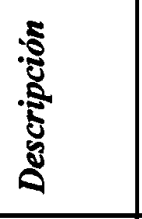 & 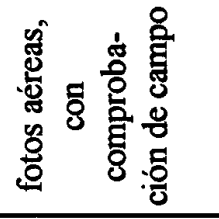 & 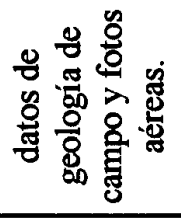 \\
\hline 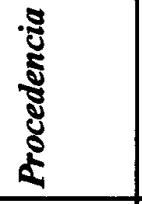 & 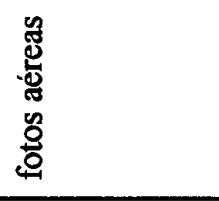 & 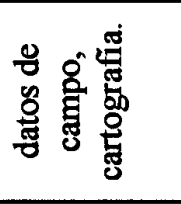 \\
\hline$\frac{8}{5}$ & & $\sqrt[5]{2}$ \\
\hline 5 & 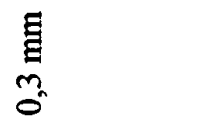 & $\begin{array}{c}E \\
\mathfrak{g} \\
\text { m } \\
\sigma^{\prime}\end{array}$ \\
\hline 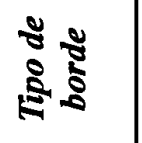 & 总号 & 导 \\
\hline 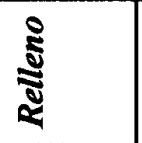 & 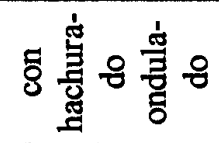 & 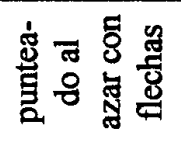 \\
\hline : & 巴્ّ & 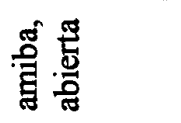 \\
\hline s & $\sim$ & $\nabla$ \\
\hline ర్ & $\sim$ & $\nabla$ \\
\hline 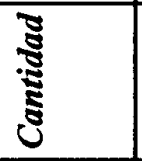 & $n$ & - \\
\hline$\frac{5}{8}$ & 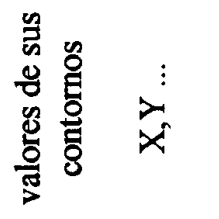 & 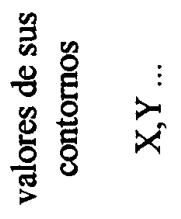 \\
\hline 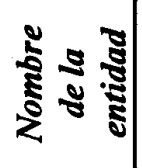 & 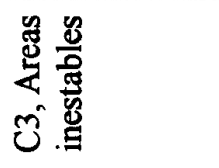 & $\begin{array}{l}\frac{1}{8} \\
\dot{0} \frac{3}{8} \\
\dot{0} \frac{y}{8}\end{array}$ \\
\hline
\end{tabular}




\begin{tabular}{|c|c|c|}
\hline 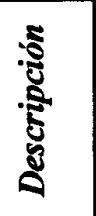 & 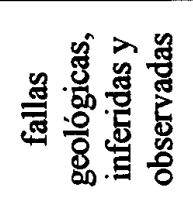 & 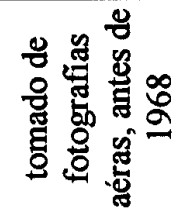 \\
\hline 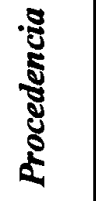 & 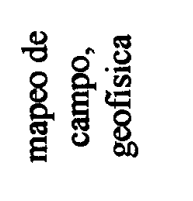 & 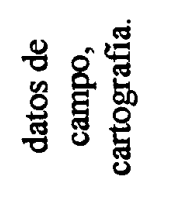 \\
\hline $\begin{array}{l}\text { है } \\
\text { है }\end{array}$ & & \\
\hline $\begin{array}{l}5 \\
5 \\
5 \\
5\end{array}$ & 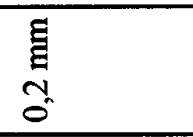 & $\frac{E}{0}$ \\
\hline$\stackrel{2}{2}$ & 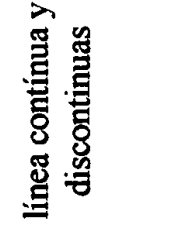 & 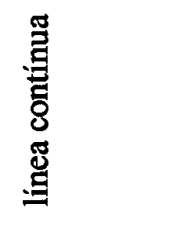 \\
\hline$\frac{5}{8}$ & $r$ & $\infty$ \\
\hline s & $\infty$ & $=$ \\
\hline 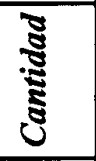 & 6 & $\rightarrow$ \\
\hline 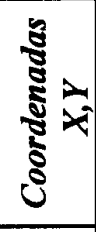 & 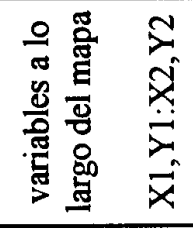 & 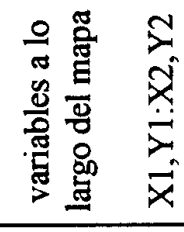 \\
\hline 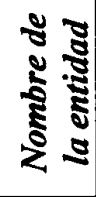 & 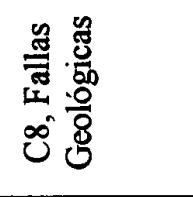 & 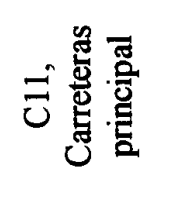 \\
\hline
\end{tabular}



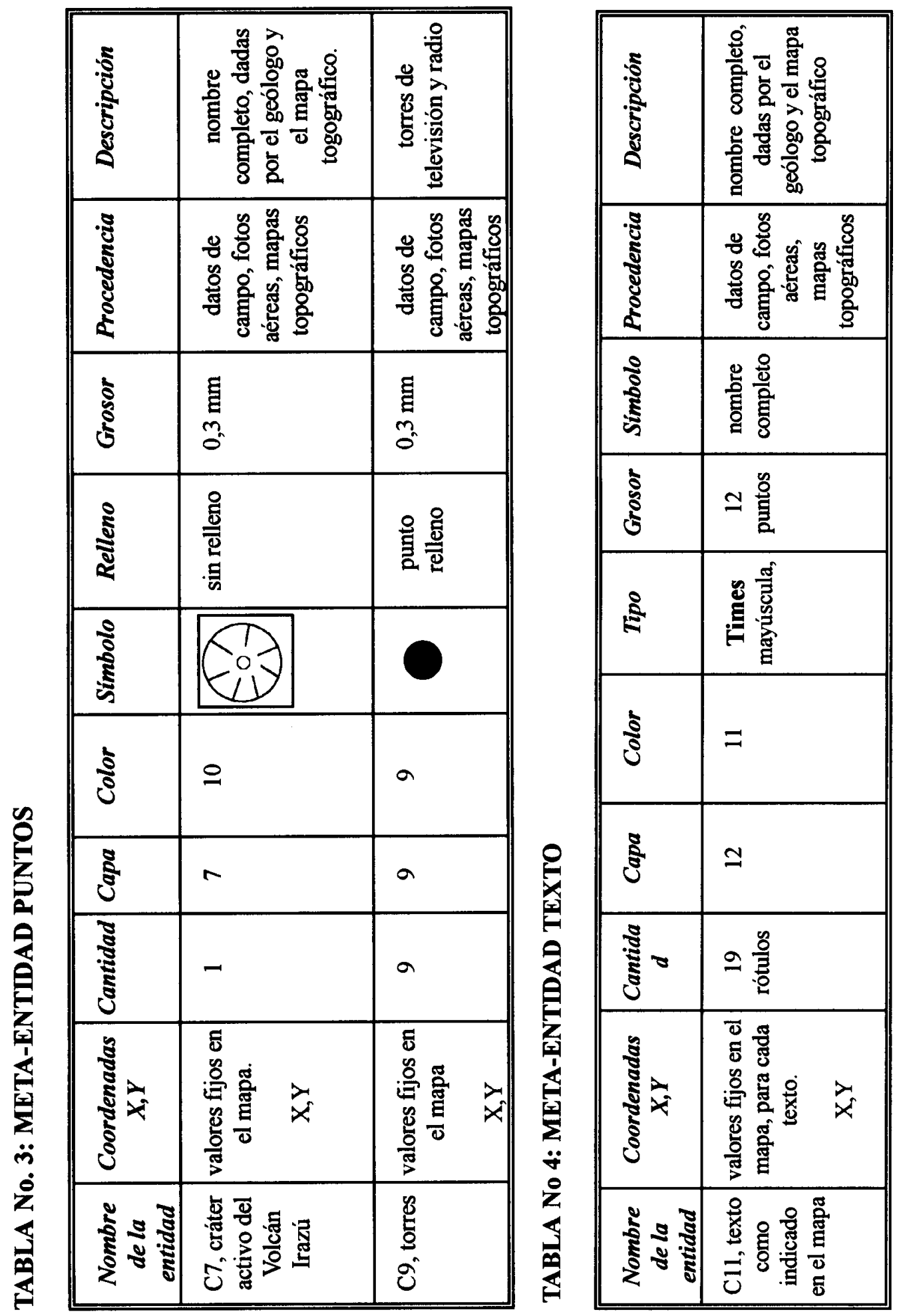


\section{EJEMPLO DE UNA REPRESENTACIÓN COMPLEJA}

Se definen como representaciones complejas aquellas en las cuales es posible subdividir el Nivel 0 en varios Subniveles 0, tantos como el usuario considere necesario. El desarrollo de tal representación podrá seguir caminos separados para cada uno de los Subniveles 0. Así, se puede llegar a un Nivel 1 separadamente, ya que cada Subnivel 0 puede tener un entorno diferente. Todos los Subniveles deben convergir en el Nivel 2, en donde se lleva a cabo la caracterización, las relaciones y la descripción (véase Fig. 2).

A continuación se desarrollará un ejemplo que representa el mapa geológico (volcánico) complejo de una vasta área de la parte central de Costa Rica (ver Fig. 7). Involucra las ciudades de Ciudad Quesada y San Ramón hacia el oeste y hasta Turrialba hacia el este. Entre los depósitos volcánicos del Cuaternario se encuentran, depósitos laháricos y fluviatiles, lavas y tefras, ignimbritas, Formación Intracañon. Los depósitos Terciarios son: basaltos alcalinos, Grupo Aguacate, rocas intrusivas y las rocas sedimentarias. Por otra parte, se localizan fallas (varios tipos), conos volcánicos (primarios y secundarios), calderas volcánicas, lagos y lagunas volcánicas y por último, ciudades. Como se observa en la figura 7 , la distribución de los rasgos lineales, puntuales y areales son complejos en este mapa.

\section{Nivel 0}

El Nivel 0 (figura 8a), representa los elementos presentes en el mapa (Fig. 7). Se ha tomado como Capa Base, los depósitos de lavas y tefras (C1) dado que esta litología es la dominante y se encuentra muy distribuida en el mapa. La mayoría de las otras unidades geológicas se localizan en una cercanía inmediata respecto a $\mathrm{C} 1$.

\section{Subniveles 0}

La representación de tales rasgos geológicos se puede hacer separando los rasgos en dos Subniveles 0. Un Subnivel 0A (Conos Volcánicos) y otro Subnivel 0B (Fallas Geológicas) (Fig. 8b y c). El diseñador puede identificar la presencia de varios Subniveles 0, cuando un mismo objeto es categorizado en varias simbologías. Por ejemplo, las fallas geológicas pueden tener dos o tres representaciones de líneas. Los rasgos puntuales pueden ser representados utilizando tres o cuatro tipos de símbolos diferentes, etc.

Estudiando el mapa geológico (Fig. 7), se observa que los rasgos lineales y puntuales, esto es, fallas geológicas y conos volcánicos respectivamente, son de dos o tres tipos. Así por ejemplo, las fallas geológicas son dibujadas como líneas continuas, líneas discontinuas y líneas dentadas, en función del tipo de falla. La simbología de los conos volcánicos principales se indica como un conjunto de líneas radiales, emanando de un centro común, estas líneas radiales no se unen. Para los conos volcánicos secundarios, su simbología es parecida a un asterisco pequeño.

\section{Nivel 1}

La representación del Nivel 1 se hará para todo el mapa geológico, esto es, el Nivel 1 estará representado por poblados secundarios, ríos más importantes y curvas de nivel cada $100 \mathrm{~m}$. Todos estos rasgos básicos estarán contenidos en un sistema externo y serán tomados de un SIG. En este caso, no es necesario continuar con los subniveles 1 , dado que todos utilizan los mismos parámetros del SIG, o sea, el mapa topográfico base (curvas de nivel, poblados, ríos, carreteras, etc., Fig. 9a.).

\section{Nivel 2}

De acuerdo a la metodología de representación, el Nivel 2 se caracteriza por la definición de las Relaciones, de la Descripción y Características.

Relaciones: Para el desarrollo de las relaciones, se ha representado el objeto C13, San José y la amenaza volcánica que la rodea (C9B), (Fig. 9b).

La figura 10, muestra las relaciones entre cada volcán y la cercanía o lejanía de las ciudades aledañas, como se indicó anteriormente. El poblado o ciudad encerrada en un cuadrado, representa, de acuerdo a los estudios geológicos y de amenazas naturales, los objetos más amenazados. 

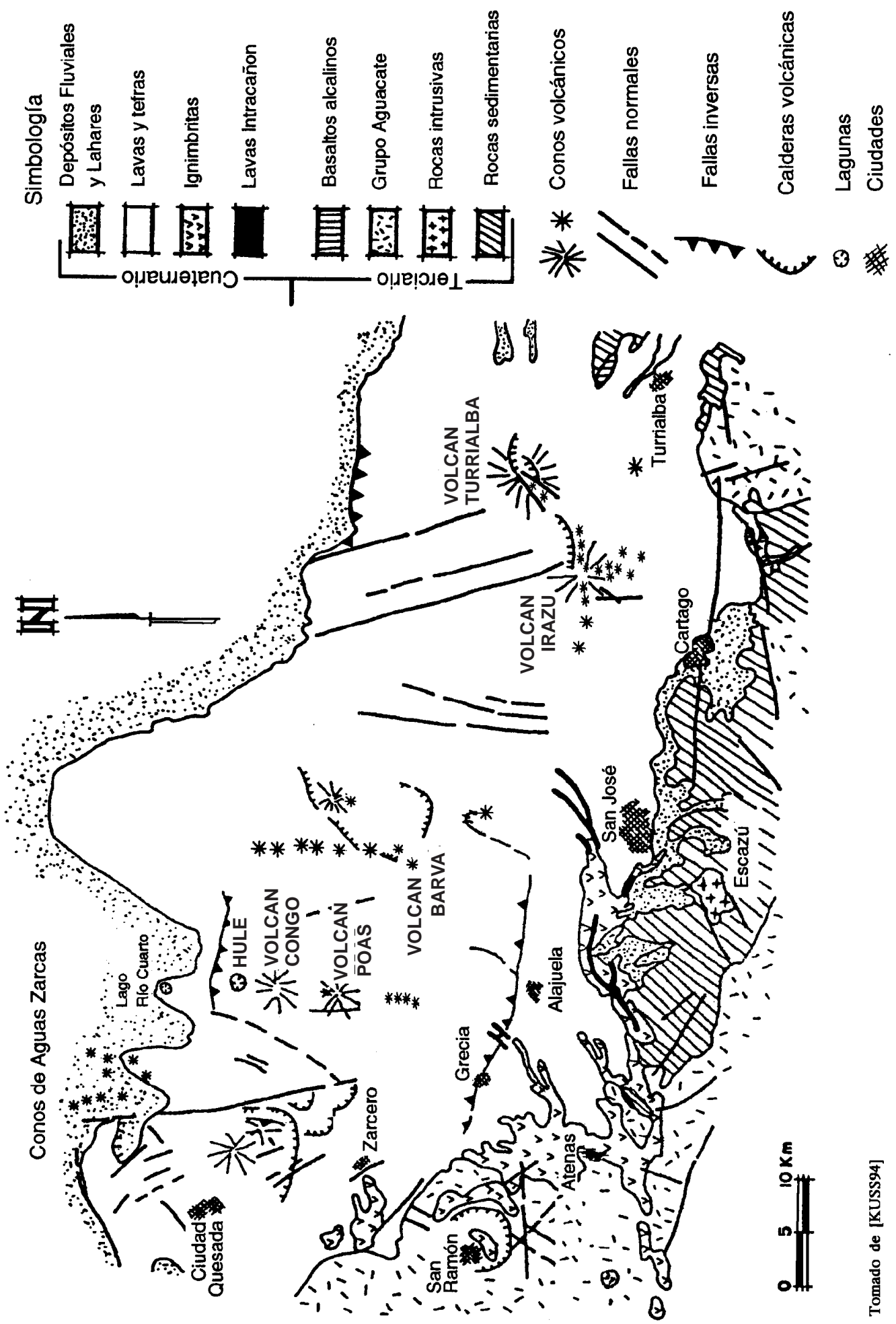
a) NIVEL 0

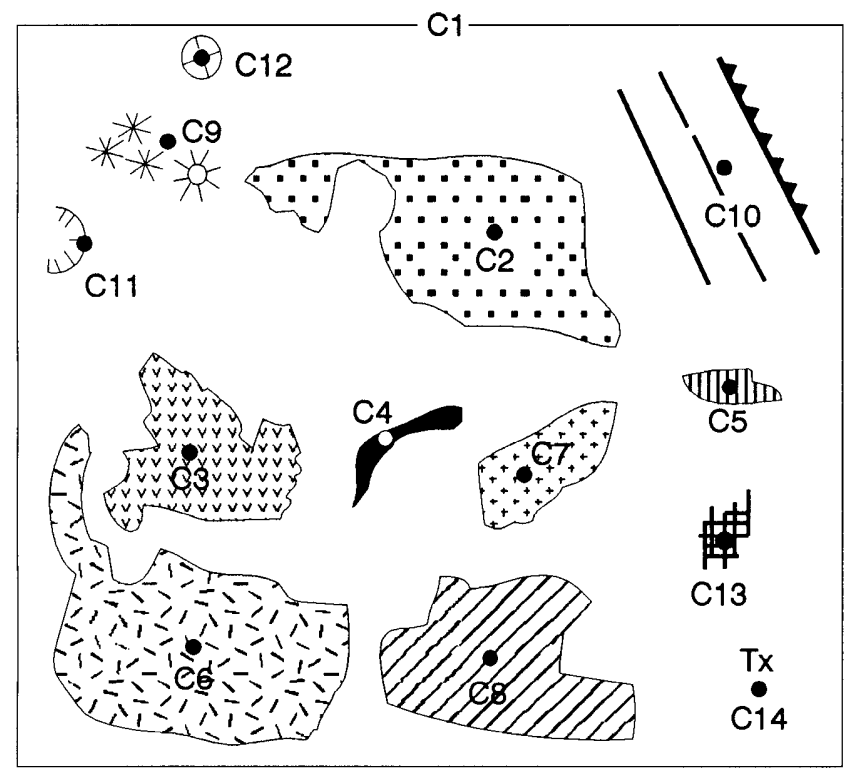

Simbología

Símbolo Capa Descripción

$\square$

C1 Lavas y tefras

$\therefore \quad$ C2 Dep. fluviales y lahares

V. C3 Ignimbritas

C4 Lavas Intracañon

[III] C5 Basaltos alkalinos

i. C6 Grupo Aguacate

[+] C7 Rocas Intrusivas

D C8 Rocas Sedimentarias

* c9 Conos Volcánicos

औr

lif C10 Fallas

E C11 Calderas

Q C12 Lagos

价13 Ciudades

Tx $\quad$ C14 Texto b)

SUBNIVEL 0 A: Conos Volcánicos

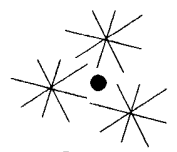

C9A

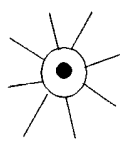

C9B c)

SUBNIVEL 0 B: Fallas Geológicas

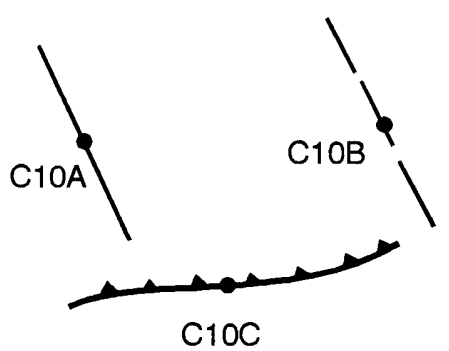

Simbología

C9A Conos volcánicos menores

C9B Conos volcánicos mayores 
a) NIVEL 1

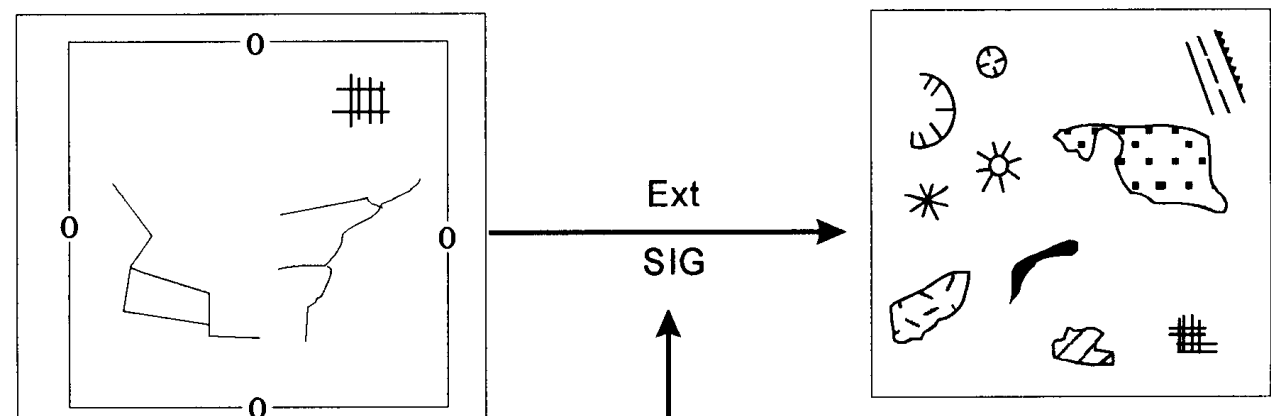

SIMBOLOGIA

$\Gamma^{\circ} 7$ Representación de curvas

$L_{0} . j$ de nivel cada $100 \mathrm{~m}$

\# Poblados, ciudades secundarias

Descripción: Mapa topográfico base 1:200,600. Archivo SiG

$\Rightarrow$ Ríos más importantes

Carreteras primarias

b) NIVEL 2

\section{Relación C13 R C9B}

\section{Volcán Barba}

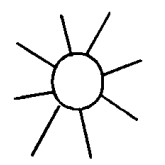

C9B<smiles>CC1(C)C(C)(C)C(C)(C)C(C)([18OH])C1(C)C</smiles>

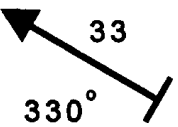

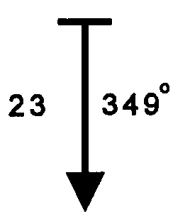

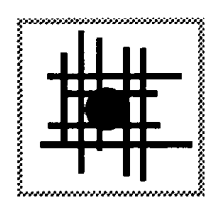

C13

an José
Volcán Irazú

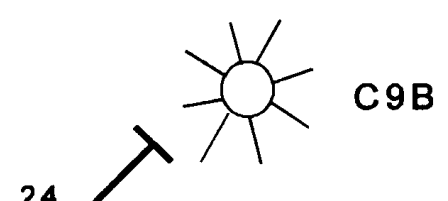

24 $78^{\circ}$

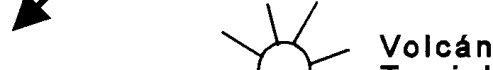<smiles>CCCCCCCC(C)C(C)CC</smiles>
Turrialba

Fig. 9. Nivel 1 y Nivel 2. a) Nivel 1, interacción de los Sistemas Externos, en este caso un SIG. b) Nivel 2, relaciones, se muestra la relación de C13 en C9B. San José está mayormente amenazada por los volcanes Irazú y Barba, dado el tipo de actividad y la dirección del viento. La definición de amenaza para el área ha sido tomado de PANIAGUA (1993 y 1994). 


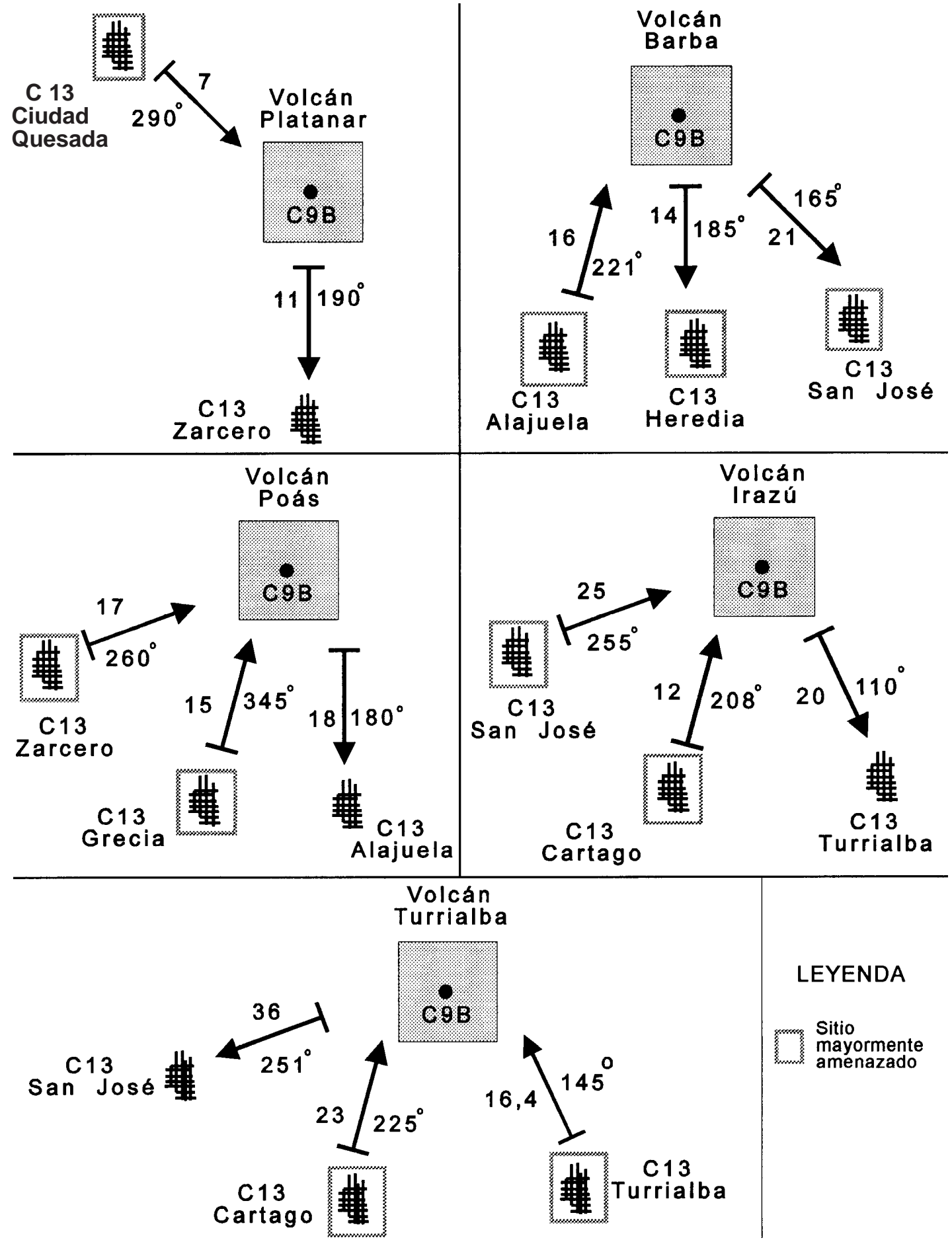

Fig. 10. Nivel 2. Descripción de relaciones. Representación de la amenaza natural, provocada por los volcanes (C9B) con respecto a las ciudades (C13). Las ciudades no amenazadas se alejan del centroide C9B. 


\section{Características y descripción.}

A continuación se describe cada objeto con detalle, este paso es el último antes de su implantación utilizando un SIG. Siguiendo con las reglas, todos los objetos presentes se agruparán en meta-entidades que se clasifican como áreas, líneas, puntos y texto. Estas tablas se relacionan con las figuras 7 y 8 .

$\begin{array}{ll}\text { Meta-entidades: } \\ \text { AREAS } & =\mathrm{C} 1 \text { hasta } \mathrm{C} 8 \\ \text { LINEAS } & =\mathrm{C} 10 \\ \text { PUNTOS } & =\mathrm{C} 9, \mathrm{C} 11 \text { hasta } \mathrm{C} 13 \\ \text { TEXTO } & =\mathrm{C} 14\end{array}$

Con estas tablas (tablas $\mathrm{N}^{\circ} 5$ a 8 ), culmina la presentación de MODAGE, dado que a partir de ellas, solo resta la implantación del proyecto utilizando el SIG de preferencia del usuario, ya sea orientado a vectores, tipo "raster" o mixto.

\section{DISCUSION}

La metodología expuesta, pretende ser simple, flexible, produce productos reusables y organiza al usuario en cuanto a la entrada de datos se refiere. Pretende ser:

Simple: porque la documentación de la información requiere de pocos pasos, siguiendo reglas bien definidas, concretas y flexibles, además está orientada al usuario final. Por otra parte, obliga al usuario a observar la complejidad de un mapa desde otro punto de vista, el cual no coincide con el de un geodesta o un geógrafo, geólogo, etc.

Flexible: porque las reglas permiten al usuario tener libertad dentro de su representación, con el fin de manipular mejor la representación de los objetos a modelar, pudiendo utilizar, por ejemplo, la simbología o notación más adecuada a sus necesidades. Por otra parte, permite al usuario desarrollar su documentación a través de niveles cada vez más complejos, que van de lo general a lo específico, hasta tener todo el proyecto estructurado para la implantación.

En lo que respecta a las amenazas naturales, la facilidad de representar a nivel esquemático las relaciones espaciales entre los objetos (Nivel 2, Relaciones o asociaciones), permite al usuario, ver de una manera simple la complejidad de éstas.
El tipo de representación de las relaciones del Nivel 2 es útil para esquematizar las amenazas (sean estas complejas o no), a partir de mapas de amenazas previamente realizados o cuando no se tienen datos previos de la zona afectada, este último caso no es más que una simple triangulación, haciendo uso de instrumentos de campo de uso común y poco sofisticados. Para estos casos, los tomadores de decisiones podrán asistirse con estos esquemas, los cuales pueden ser posteriormente, sustituidos por los mapas formales de campo.

Productos reusables: La documentación generada al final del Nivel 2 puede servir como consulta para otros proyectos SIG durante o posterior a la ejecución de determinado proyecto. Las referencias a otros sistemas, datos de entradas, productos obtenidos, entidades trabajadas y sus atributos, etc., son debidamente documentados.

Organización del usuario: Todo mapa (topográfico, geológico, vulcanológico, etc.), presenta grados de complejidad distintos y usualmente elevados, dado que se sobreponen diferentes niveles de objetos con una semántica propia entre sí, pero que forman una unidad. Tal complejidad puede ser dificil de manejar, hasta imposible si los proyectos SIGs son grandes. Los niveles propuestos por MODAGE obligan al usuario a compenetrarse con el proyecto, a ordenar las entidades y a entender los diversos objetos involucrados espacialmente, así como sus relaciones y atributos. Por tanto, el usuario llega a dominar el proyecto SIG antes de su implantación.

MODAGE, Toma de Decisiones y las Fases del "Antes" y del "Durante" de un desastre

En PIEPTEA (1987) se clasifica la toma de decisiones dentro de una organización de acuerdo a la estructura jerárquica y a los objetivos de dicha organización.

Los criterios en relación al proceso de la toma de decisiones, son:

1) Grado de estructuración de la decisión.

2) Nivel de la actividad administrativa.

3) Grado de incertidumbre.

4) Fuente de información usada.

En una organización cualquiera, el grado de estructura de los problemas cambia sistemáticamente al viajar desde los niveles inferiores 


\begin{tabular}{|c|c|c|c|}
\hline 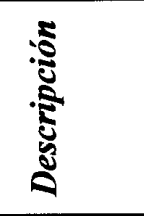 & 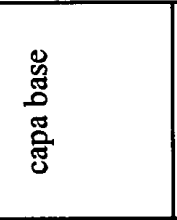 & 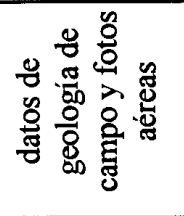 & 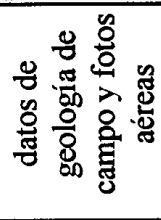 \\
\hline 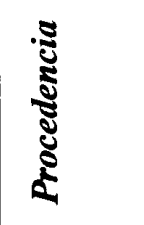 & 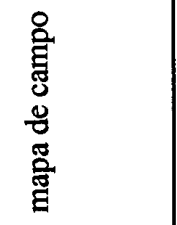 & 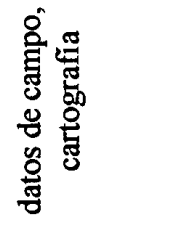 & 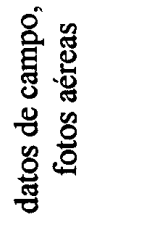 \\
\hline हैँ & 을 & 60 & \\
\hline $\begin{array}{c}5 \\
5 \\
5 \\
5\end{array}$ & 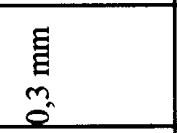 & $\begin{array}{l}E \\
E \\
e^{2} \\
\end{array}$ & 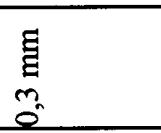 \\
\hline 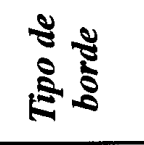 & 名 & 导兽 & 兽 \\
\hline ֻั๊ & 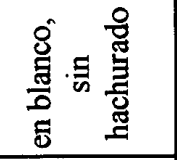 & 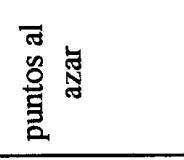 & 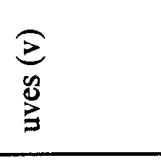 \\
\hline$\stackrel{5}{5}$ & 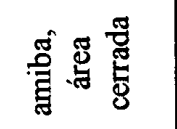 & 总 & 苞 \\
\hline 8 & - & $N$ & $m$ \\
\hline हू & - & N & $m$ \\
\hline 胥 & - & $N$ & $N$ \\
\hline 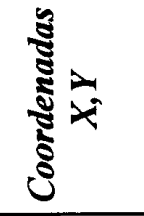 & 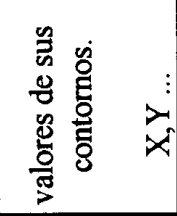 & 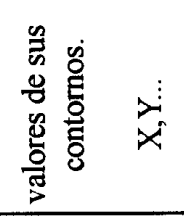 & 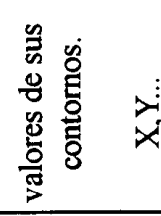 \\
\hline 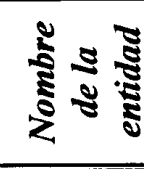 & 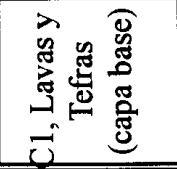 & 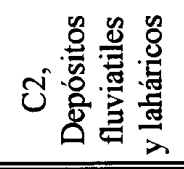 & 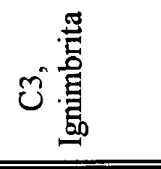 \\
\hline
\end{tabular}




\begin{tabular}{|c|c|c|c|c|}
\hline 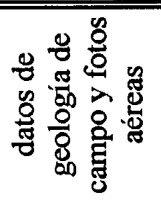 & 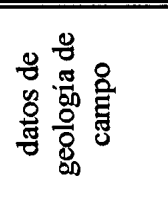 & 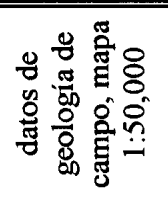 & 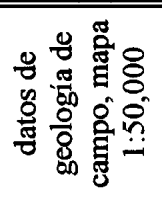 & 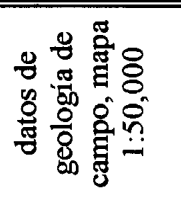 \\
\hline 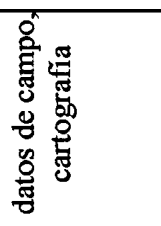 & 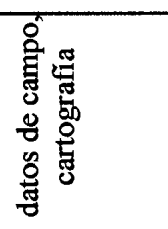 & 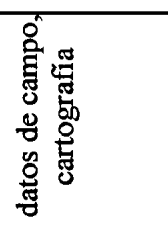 & 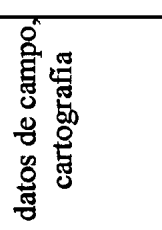 & 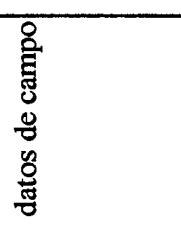 \\
\hline & & ${ }^{\prime}, 1$ & $+++^{+}$ & \\
\hline 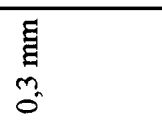 & 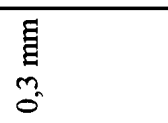 & $\begin{array}{l}\xi \\
\text { E } \\
m \\
m \\
0\end{array}$ & 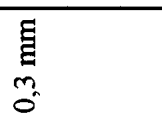 & 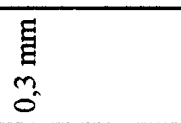 \\
\hline 鸹 & 驾 & 导 & 嵒 & 导䓵 \\
\hline 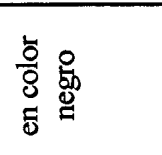 & 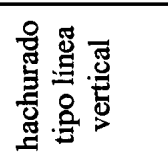 & 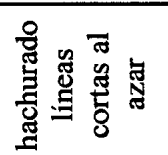 & 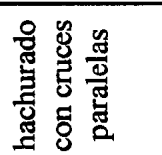 & 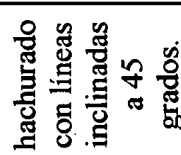 \\
\hline 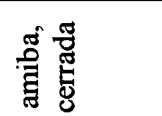 & 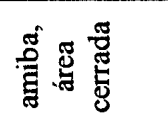 & 苛 & 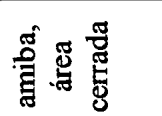 & 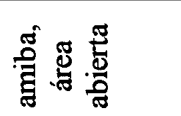 \\
\hline$\theta$ & $n$ & 6 & $n$ & $\infty$ \\
\hline$\theta$ & $n$ & 6 & $n$ & $\infty$ \\
\hline$\nabla$ & - & - & - & - \\
\hline 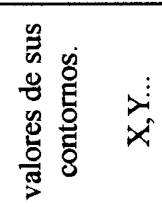 & 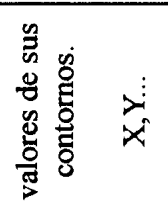 & 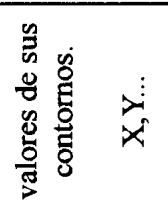 & 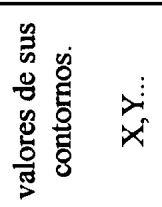 & 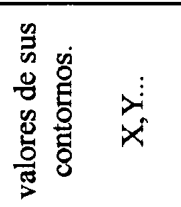 \\
\hline 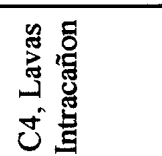 & 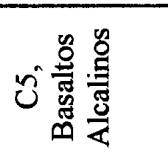 & 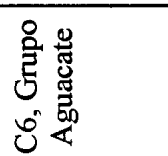 & 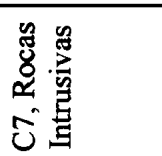 & 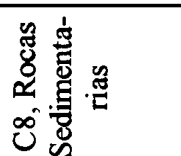 \\
\hline
\end{tabular}




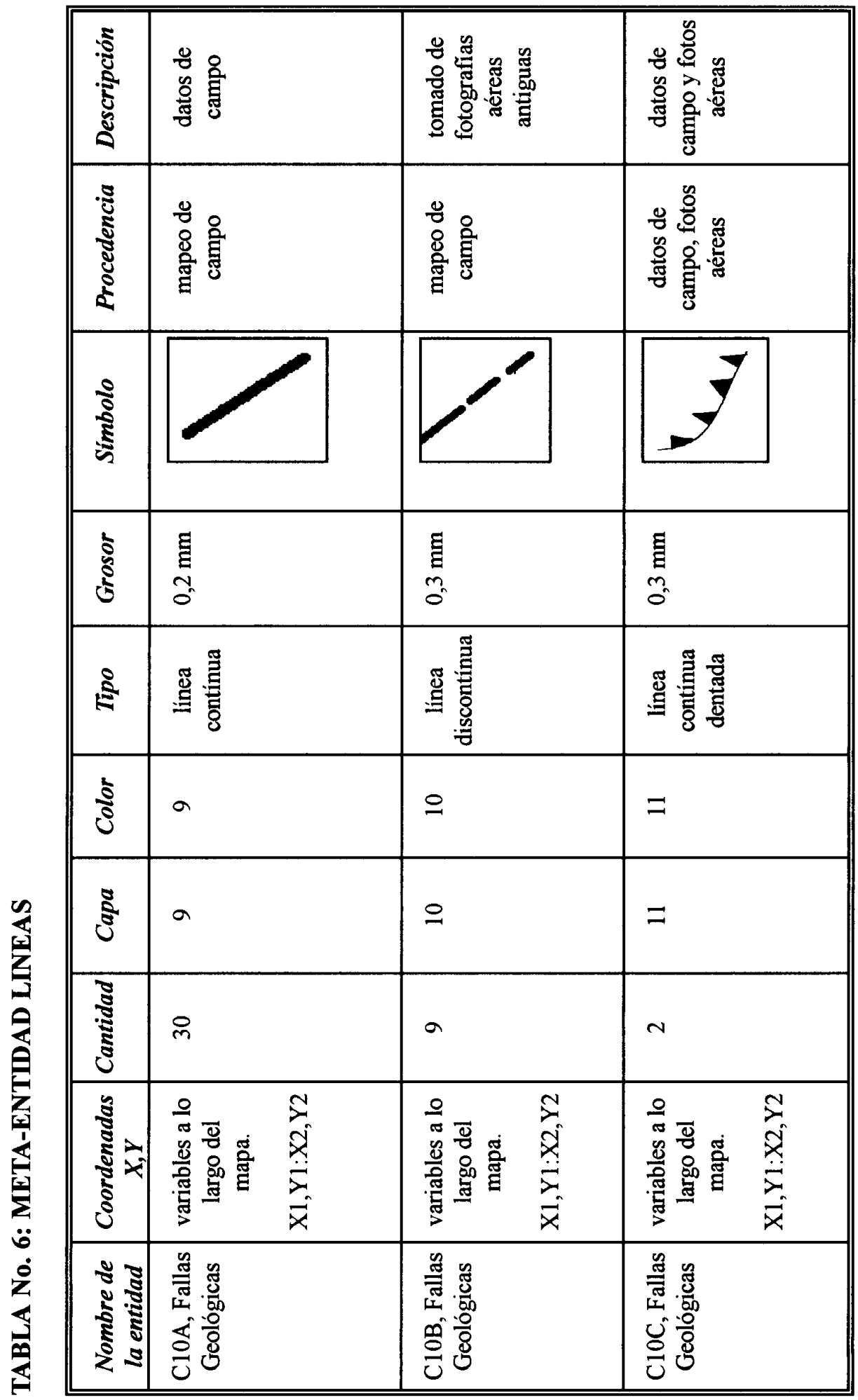




\begin{tabular}{|c|c|c|c|c|c|}
\hline 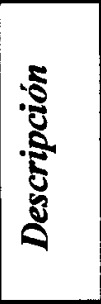 & 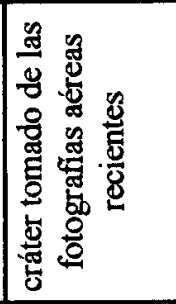 & 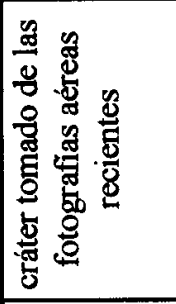 & 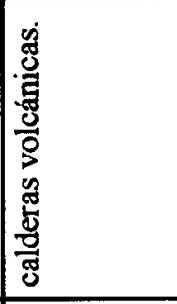 & 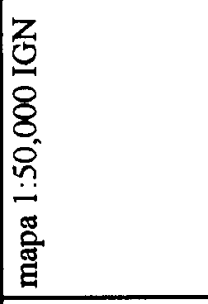 & 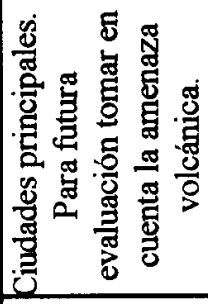 \\
\hline 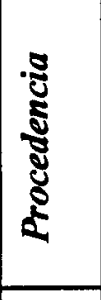 & 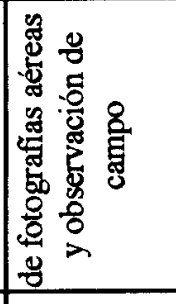 & 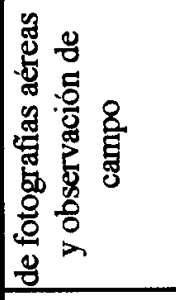 & 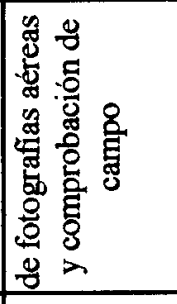 & 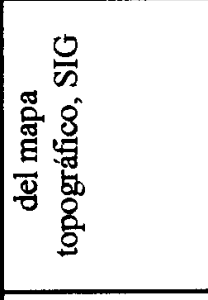 & 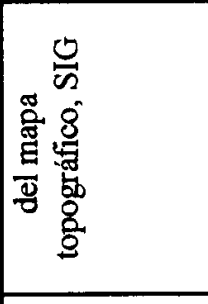 \\
\hline है & & & & & 册 \\
\hline Еัँ & 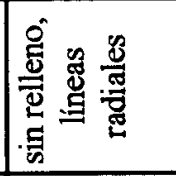 & 总 & 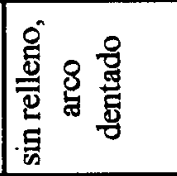 & 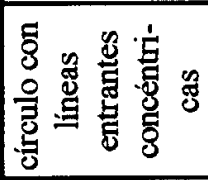 & 这 \\
\hline 5 & 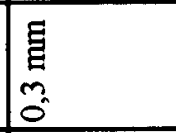 & $\begin{array}{l}E \\
\text { E⿱ } \\
\text { m. } \\
\text { on } \\
\end{array}$ & \begin{tabular}{|l}
$\mathbf{g}$ \\
$\mathrm{m}$ \\
$\mathrm{m}$ \\
0
\end{tabular} & 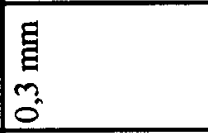 & \begin{tabular}{|c|} 
\\
E⿱ \\
$m$ \\
0 \\
0
\end{tabular} \\
\hline$\frac{5}{8}$ & $\simeq$ & $m$ & \pm & $\cong$ & \pm \\
\hline s. & $\simeq$ & $m$ & $=$ & $\cong$ & \pm \\
\hline 胥 & 0 & $F$ & $\infty$ & a & $a$ \\
\hline 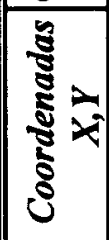 & 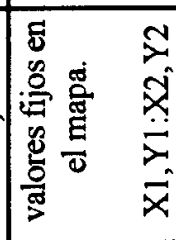 & 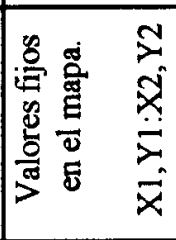 & 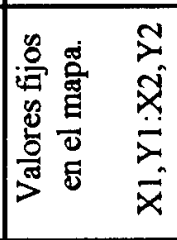 & 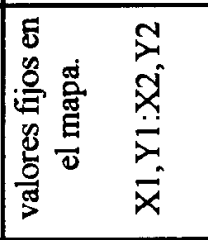 & 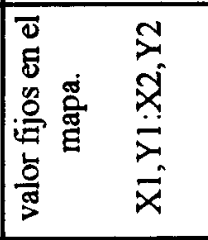 \\
\hline 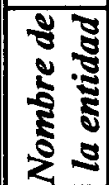 & 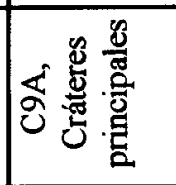 & 窇 & ప் & 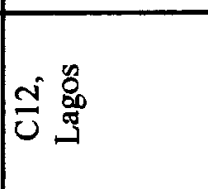 & 兽 \\
\hline
\end{tabular}




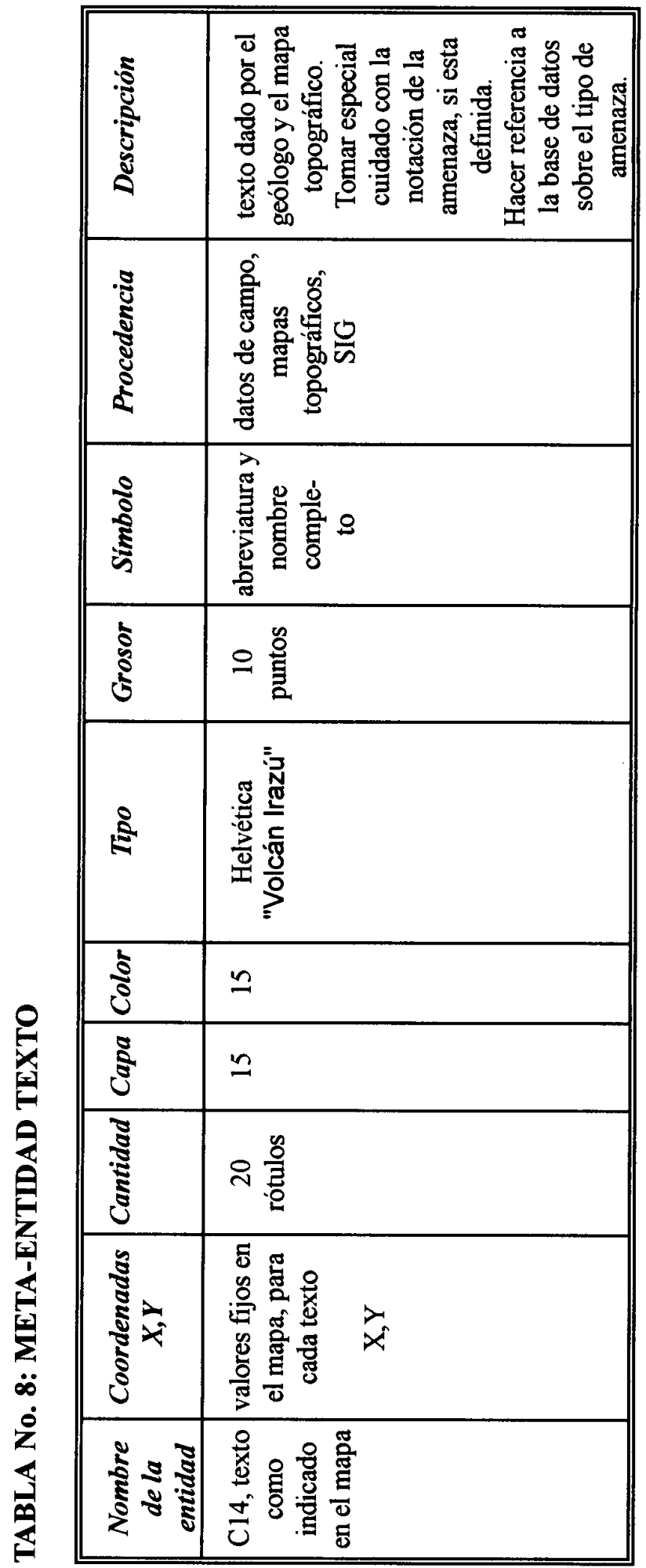


hacia los niveles altos de la estructura jerárquica. En el nivel de supervisor las decisiones son muy estructuradas, en la mitad de la jerarquía, las decisiones llegan a ser tanto estructuradas como semiestructuradas. Por último, las decisiones muy poco estructuradas dominan en los niveles altos de las organizaciones.

Basado en lo anterior, en PIEPTEA (1987) se clasifica la toma de decisiones en tres tipos:

1) Clase 1: Decisiones altamente estructuradas, decisiones tomadas con certidumbre, típicas de los supervisores de los niveles inferiores de la jerarquía organizacional. El tipo de información utilizada es interna, propia de la organización.

2) Clase 2: Decisiones semiestructuradas, tomadas por los mandos medios y altos de la administración. Estas decisiones son tomadas tanto en ambientes de certidumbre como de incertidumbre. El tipo de información usada puede ser interna y externa a la organización.

3) Clase 3: Decisiones muy poco estructuradas, dominando un ambiente de incertidumbre. Realizadas por los jerarcas de la organización, usando información tanto interna como externa a la organización.

Por su parte, en DROR, (1988) se señala que la toma de decisiones bajo condiciones de desastre, envuelve un juego difuso en el sentido de que las decisiones deben ser hechas de cara a la incertidumbre y las reglas del juego son desconocidas y a menudo indeterminadas y erráticas.

ANTHONY en (PIEPTEA, 1987), indica que los niveles de la actividad administrativa se clasifican en tres:

1) Planeamiento estratégico (PE), se refiere a las decisiones tomadas sobre los objetivos de la organización, los recursos utilizados y las políticas para administrar estos recursos (mandos altos, ejecutivos)

2) Control administrativo (CA), asegura que los recursos sean utilizados eficientemente para atender los objetivos de la organización (mandos medios, ejecutivos medios).

3) Control operativo (CO), asegura que las tareas sean ejecutadas apropiadamente. (mandos bajos, supervisores).
La prevención incluye el desarrollo de escenarios de desastres potenciales, mapeo de amenazas, creación y regulación de leyes, restricciones en el uso de la tierra, la aplicación de las medidas estructurales de tipo ingenieril, etc. Por lo tanto, los tres niveles anteriores de la actividad administrativa pueden llevarse a cabo.

La fase de mitigación, comprende ayudas y estrategias. Todas aquellas acciones tomadas para minimizar los efectos de un desastre pueden verse como Planificación Estratégica (Estrategia Gerencial) orientada a resolver problemas.

La etapa del "Durante" hace que las resoluciones lleven consigo la toma de decisiones rápidas y a diferencia de la prevención, estas resoluciones normalmente deben tomarse contra el tiempo.

Respecto a eso, en DROR (1988) se señala que los eventos ocurren muy rápido y requieren de una respuesta inmediata. Por otra parte, pueden engendrar implicaciones de largo alcance. La toma de decisiones bajo condiciones de desastre puede actuar bajo una simple, repetida y continua calamidad.

En algunos párrafos anteriores, se indicó que la metodología propuesta (MODAGE), puede asistir también a la toma de decisiones, tanto estructuradas como semiestructuradas. En este apartado, se discutirá las decisiones estructuradas, semiestructuradas a poco estructuradas en relación con MODAGE.

\section{a) Decisiones altamente estructuradas}

Al definirse e implantarse una metodología de trabajo, estandarizando y documentando cada proyecto SIG, los supervisores tienen una valiosa ayuda en la ejecución de determinadas tareas cuando se viven ambientes de desastre, dado que todo el personal involucrado sabe qué hacer, sabe dónde están los archivos y la documentación correspondiente, sabe recoger la información, procesarla, registrarla, graficarla y presentarla. Es decir, se actúa con decisiones altamente estructuradas, tomadas con certidumbre. Así, el tiempo de ejecución se reduce y la respuesta tiende a ser más rápida.

b) Decisiones semiestructuradas a poco estructuradas (tomadas con grados de incertidumbre)

Al definirse las relaciones del Nivel 2, sobre todo en casos de emergencia, se define 
esquemáticamente la situación en el sitio de amenaza o de desastre, por lo tanto, los mandos medios o jerarcas de la organización pueden tomar sus decisiones partiendo de tales esquemas (ubicados espacialmente), teniendo como punto de partida las relaciones del Nivel 2. De este modo, la incertidumbre durante los primeros estadios en la etapa de Respuesta tiende a disminuir.

\section{MODAGE y la Toma de Decisiones}

DROR (1988), indica que los procesos y procedimientos de decisión son el núcleo de la toma de decisiones bajo condiciones de desastre y por tanto, requieren de una cuidadosa consideración y preparación. Entre esos procedimientos y procesos sugiere:

Estimación contínua y "retratos" actualizados de la situación de desastre. Esta información es buena si se usan computadoras gráficas interactivas y sistemas de información usados simultáneamente, tanto por el tomador de decisiones así como por las estaciones de análisis.

- Especial atención debe dársele a las condiciones de incertidumbre. Todos los módulos de análisis de políticas y programas usados, necesitan ser sensitivos a la incertidumbre con el fin de evitar métodos inapropiados.

Respecto a lo anterior y dado que los primeros estadios de la fase del "Durante", se caracterizan por ser saturados de incertidumbre, las relaciones definidas en el Nivel 2 pueden ayudar a especificar mejor las relaciones espaciales entre los objetos, por lo tanto, a reducir el nivel de incertidumbre. Si un observador ubicado en el área de desastre describe relaciones espaciales apropiadamente, por ejemplo, vía radio, es posible dibujar las relaciones. Con ello, el tomador de decisiones puede ubicarse espacialmente con respecto a la amenaza, aunque sea tan solo de manera esquemática. Por otra parte, el especialista en cómputo puede introducirlo al computador para que este ubique espacialmente, de manera relativa, los objetos descritos por el observador de campo. Posteriormente, tales esquemas pueden ser sustituidos por mapas formales de campo, levantados directamente en el área de desastre.
La comparación posterior, entre el mapa de campo y los esquemas de relaciones servirá como medio de autoevaluación, en cuanto a la descripción de relaciones espaciales entre objetos se refiere.

Por último, MODAGE, puede asistir directa $\mathrm{o}$ indirectamente, en la toma de decisiones durante las fases del "Antes" (Prevención, Mitigación) y del "Durante" (Respuesta) de un Desastre Natural, especialmente en las Clase 2 y Clase 3 de PIEPTEA (1987), con énfasis en ésta última, en donde privan las decisiones poco estructuradas y existe una dominancia de un ambiente de incertidumbre, por otra parte, cumple con lo dispuesto por DROR (1988). En cuanto a las actividades, MODAGE asistirá básicamente, en las fases de Planeamiento Estratégico y Control Administrativo.

\section{CONCLUSIONES}

La Metodología para el Modelaje de Datos Geográficos, está diseñada para ser utilizada con la tecnología SIG y se orienta al usuario final, esto es, usuarios con poca o nada de experiencia en proyectos SIG. Básicamente ayuda a la documentación, definición de entidades y atributos, haciendo uso de la generalización. Por último, mediante la definción de relaciones espaciales asiste a la toma de decisiones tanto estructuradas como semiestructuradas.

MODAGE, define tres niveles lógicos, permitiendo al usuario abstraerse de la complejidad que presentan los mapas actualmente, para ello, MODAGE define tres niveles:

Nivel 0, es el nivel básico de representación, en donde el diseñador solo mostrará los elementos gráficos presentes y sus centroides, los centros del objeto gráfico.

Nivel 1, se mostrarán las influencias externas de otros sistemas, es decir, su entorno. Se representarán los sistemas que interactúan con el proyecto a modelar.

Nivel 2, se definen las características de los objetos y sus relaciones espaciales con otros objetos, la importancia que estos tienen respecto a las amenazas, crisis o desastres naturales. Por último, se plantea la descripción detallada de cada objeto modelado, por medio de un diccionario de datos. Con el Nivel 2, se concluye la documentación del sistema, listo para ser implantado utilizando un SIG. 


\section{AGRADECIMIENTOS}

Se agradece profundamente al Dr. Carlos Gonzáles (Director de la Maestría en Computación, Instituto Tecnológico de Costa Rica, TEC) por la supervisión esmerada durante la realización de la presente investigación. A los master Timothy Robinson (Laboratorio de Sistema de Información Geográfica, Universidad Nacional, UNA), a Sergio Paniagua (Director de la Escuela Centroamericana de Geología, UCR) por las revisones, sugerencias, ideas y apoyo, durante la realización del presente trabajo. Asimismo, agradezco al Lic. Mario Garcia, informático, por mostrar gran interés al leer y corregir el manuscrito inicial.

A las siguientes personas: Sr. Marlon Molina (Informático), Sr. David Aguilar (Sistemas Analíticos), Sr. Lorenzo Jiménez (Laboratorio Sistema de Información Geográfico, Universidad Nacional), mi reconocimiento por la información facilitada, disponibilidad e interés durante la realización de la presenta investigación. A todas aquellas personas que de alguna u otra manera ayudaron en la presente investigación.

\section{REFERENCIAS}

DROR, L.,1988: Decision making under Disaster Conditions. - En: Comfort, L (ed.),
Managing Disaster (Strategies and Policy Perspectives): 255-273; Duke Unv. Press.

PANIAGUA, S.P.; SALAZAR, L.G.; KUSSMAUL, S.; MONGE A; OBANDO, L. G.; BURGOS A; FERNÁNDEZ, M; 1993: Amenaza y Estimación Económica básica de la Vulnerabilidad material asociada al Volcán Irazú, Cordillera Central, Costa Rica. - Escuela. Centroamericana. de Geología. UCR- CEPREDENAC, (Informe técnico final), 80 págs.

PANIAGUA, S. P.; 1994: Amenaza Volcánica. En: Denyer P. \& Kussmaul S. (comp). Atlas Geológico del Gran Area Metropolitana, ITC: 221-231.

PIEPTEA, D. \& ANDERSON, E., 1987: Price and Value of Decision Support Systems. MIS QUARTERLY: 515-527, December 1987.

KUSSMAUL, S; TOURNON, J.; ALVARADO, G.; 1994: Evolution of the Neogene to Quaternary Igneous Rocks of Costa Rica. Profil ,7: 97-123. 